\title{
Nonstoichiometry and Properties of SnTe Semiconductor Phase of Variable Composition
}

\author{
Elena Rogacheva \\ National Technical University "Kharkov Polytechnic Institute" \\ Ukraine
}

\section{Introduction}

According to the modern views, all semiconductor compounds have a homogeneity region (HR), i.e. represent phases of variable composition. The existence of thermodynamically equilibrium deviation from stoichiometry in a chemical compound is connected with a decrease in the crystal free energy under introduction of nonstoichiometric defects (NSD) whose appearance leads to a growth in configurational entropy. The location and size of HR depend on the ratio of the formation energies for different types of defects. The stoichiometric composition may lie inside or outside HR (which corresponds to two-sided and one-sided HR, respectively). Changing properties through deviation from stoichiometry is one of the most important methods of controlling properties of semiconductors.

A large group of semiconductor phases of variable composition are so-called heavily nonstoichiometric (NS) phases with wide HR and high concentrations of NSD. With regard to these phases, there arise a number of questions related to the defect interaction and its effect on the crystal structure, energy band spectrum, and physical properties. The fundamental concepts of statistical thermodynamics of point defects are valid on condition that defects can be considered as noninteracting particles and their distribution has a purely statistical character (Kröger, 1973). That is why the Wagner-Schottky theory (Wagner \& Schottky, 1930) can provide satisfactory description only for the compounds with a very narrow HR, whereas for phases with a wide HR it is necessary to take into consideration the defect interaction (Coulomb, deformational, etc.), which can lead to the defect ordering. At present, there is extensive experimental material confirming the possibility of a short- and long-range ordering of NSD in wide HR (Collongues, 1974, 1993; Rabenau, 1975). An intermediate character of chemical bond in semiconductors and the appearance of free charge carriers induced by nonstoichiometry determine a great variety of possible mechanisms of nonstoichiometry. However, problems related to properties of heavily NS phases practically have not been studied. Physical phenomena accompanying significant deviations from stoichiometry are similar to phenomena taking place under heavy doping; the difference consists in the fact that under deviation from stoichiometry, the role of impurity atoms (IA) is played by NSD.

IV-VI binary semiconductors are very convenient objects for studying fundamental problems of heavily NS phases because they include compounds with wide HR (for example, SnTe). Besides, these compounds are of practical importance too, as they are 
widely applied in thermoelectricity, optoelectronics, IR-devices and other fields of science and technology (Nimtz \& Schlicht, 1985; Gornik et al., 1982; Ravich et al, 1970).

In this chapter, an overview and analysis of our experimental and theoretical data on the crystal structure, mechanical, thermal, galvanomagnetic and thermoelectric properties vs. composition of the one-sided binary semiconductor phase SnTe are given together with the results obtained by other authors. The chapter consists of three sections. Section 1 contains the results of the studies of the SnTe HR boundaries at different temperatures and the concentration dependences of properties determined by the crystal lattice dynamics and electronic subsystem of the crystal in the SnTe HR. We were first who observed peculiarities in the property-composition dependences at the composition corresponding to the maximum in the melting curves. Using the percolation theory approach, we attributed the peculiarities to the transition from weak to heavy self-doping within the HR and determined compositions optimal for long-range ordering. We proposed a principally new model of the SnTe band structure, which takes into consideration a high concentration of NSD. In section 2, the results of the study of phase transitions in SnTe bulk crystals and thin films are given. New phase transitions at a high degree of deviation from stoichiometry were detected and attributed to the processes of vacancies redistribution. The influence of kinetic factors on the temperature dependences of the properties and the character of relaxation processes was considered. Section 3 deals with the peculiarities of SnTe doping. Some new physical phenomena connected with simultaneous presence of NSD and IA are established. It is shown that the presence of cation vacancies causes a new mechanism of impurity solubility associated with filling of NS vacancies. The influence of IA on equilibrium of NSD in SnTe is considered. Recharging of IA with variable valence in the presence of the NS vacancies is revealed. It is suggested that the above mentioned phenomena are common for heavily NS phases and should be taken into account when developing semiconductor materials for different applications.

\section{Concentration dependences of properties in the SnTe homogeneity region}

SnTe is a congruently melting semiconductor compound formed in the Sn-Te system at 1063 $\mathrm{K}$ (Fig. 1,a), which can crystallize in two structures: cubic $\beta$ (space group Fm3m) and rhombohedral a (R3m) (Abrikosov \& Shelimova, 1975; Merzhanov, 1988). SnTe undergoes a ferroelectric phase transition (FPT) $\beta \rightarrow \alpha$ at a temperature $\mathrm{T}_{\mathrm{C}} \sim 100 \mathrm{~K}$ (Littlewood, 1982,1984; Suski, 1985). Chemical bonds in SnTe are of ionic-covalent-metallic character. It is assumed that the dominant role in the formation of covalent bonds is played by atomic p-orbitals. The difference in electronegativity of $\mathrm{Sn}$ and Te atoms determines the appearance of ionic component of chemical bond, and a high concentration of charge carriers due to deviation from stoichiometry leads to the existence of a metallic component (Littlewood, 1982,1984).

\subsection{Homogeneity region and defect structure of SnTe}

The HR of SnTe was studied in a number of works (Brebrick, 1963,1964,1967,1971; Maselsky\&Lubell, 1963; Shelimova\& Abrikosov, 1964). The maximum extension of the HR $(50,1-50,9$ at. $\% \mathrm{Te})$ is at $673 \mathrm{~K}$; the maximum in the melting curves corresponds to 50.4 at. \% $\mathrm{Te}\left(\mathrm{Sn}_{0.984} \mathrm{Te}\right)$. It was found that the unit cell parameter decreases linearly with increasing Te content and that the predominant defects are cation vacancies. SnTe always exhibits $p$-type conductivity and has a high hole concentration $p$, which increases with increasing Te content from $p=2 \cdot 10^{26} \mathrm{~m}^{-3}$ up to $p=1.5 \cdot 10^{27} \mathrm{~m}^{-3}$ at $300 \mathrm{~K}$. Most experimental data and calculations 
(Akchurin\& Ufimtsev, 1979; Lin et al., 1986; Kattner et al., 1985; Logachev \& Moyzhes, 1970) on the SnTe HR are related to the temperatures higher than the eutectic temperature in the Sn-Te system ( $678 \mathrm{~K})$.

We determined the boundaries of the SnTe HR at 450 - 820 K (Rogacheva et al, 1986,1991a) using a complex of different methods, in particular microstructural and X-ray diffraction (XRD) methods, measurements of microhardness $H$, Seebeck coefficient $S$, electrical conductivity $\sigma$ and other properties. The samples were subjected to different types of heat treatment (HT), specifically, annealed at $820 \mathrm{~K}$ for 500 hours (HT1), at $670 \mathrm{~K}$ for 500 hours (HT2), at $570 \mathrm{~K}$ for 2100 hours (HT3), at $450 \mathrm{~K}$ for 4200 hours (HT4), and then quenched in water. It is seen from Fig. 2,a that the unit cell parameter $a$ decreases linearly within the HR, and as the temperature of annealing decreases, the SnTe HR narrows. In Fig. 1,b, the HR of SnTe is plotted on the basis of our data and data of other authors. It is seen that with decreasing temperature, the boundaries of the SnTe HR are shifted to the composition of $\mathrm{Sn}_{0,984} \mathrm{Te}$. This is rather interesting fact, as usually in NS phases at $\mathrm{T} \rightarrow 0$ the phase composition corresponds to the stoichiometric one. One can suggest that $\mathrm{SnTe}$ remains onesided phase in the entire temperature range. Let us call such phase "genuine nonstoichiometric". The existence of such phases can be connected with a decrease in the free energy due to the formation of charge carriers (Korzhuev, 1985) or due to the formation of a superstructure of defects.

Under introduction of excess Te into the SnTe crystal the following types of the defect formation are possible: 1) Te atoms are built into the anion sublattice causing the appearance of the cation vacancies; 2) Te atoms are embedded in the tetrahedral interstitial sites; the probability of this process is very low because it leads to a significant deformation of the crystal lattice; 3) Te atoms occupy positions of Sn atoms (antistructural defects) creating vacancies in the anion sublattice or partially filling the existing cation vacancies. In IV-VI compounds, the energy of the anion vacancy formation significantly exceeds that for the cation vacancy. That is why the predominant defects in SnTe are cation vacancies.

The presence of the maxima in liquidus and solidus curves at 50.4 at.\% Te indicates the existence of a composition with maximum thermal and thermodynamic stability within the SnTe HR. One can expect that in the concentration dependences of properties there must be peculiarities corresponding to the maxima in the melting curves. To verify this suggestion, we carried out detailed studies of the concentration dependences of properties within the SnTe HR.

\subsection{Static distortions of the crystal lattice caused by nonstoichiometric vacancies}

The presence of point defects causes geometrical distortion of the crystal lattice as a result of displacement of the atoms (ions) surrounding a defect. A rigorous solution of the problem of the determination of atom displacements in the vicinity of a defect is complicated (Maradudin, 1968; Lanno \& Burguen, 1984; Burguen\&Lanno, 1985). Under the assumption of elastic continuous medium, the displacement field around a vacancy is spherically symmetrical, and the displacement diminishes proportionally to $r^{-3}$, where $r$ is the distance from the defect. As a result of the displacement of Te atoms surrounding a vacancy, the distance between them decreases, which leads to a decrease in the SnTe unit cell parameter. From the concentration dependence of $a$ in the SnTe HR, one can estimate the 
crystalochemical radius of vacancies: $r_{\mathrm{V}}=0.06 \pm 0.005 \mathrm{~nm}$. It is seen that the vacancy radius is $0.5-0.6$ of the ionic radius of $S n$, and the vacancy volume is $0.1-0.3$ of the cation volume. It should be noted that in diamond-like lattices the volume of vacancies amounts to $\sim 0,8$ of the atom volume and in FCC-lattices with metallic bonds it is 0,5-0,6 of the atom volume (Gorelik \& Dashevskii, 1988). Thus, in comparison with vacancies of the above mentioned types, NS vacancies have much smaller volume, which indicates significant static displacements of Te atoms near a vacancy. Such considerable distortions in the crystal lattice in the vicinity of vacancies under the condition of their random distribution must lead to an increase in the degree of anharmonicity of atom vibrations and other effects. This, in turn, can stimulate redistribution of vacancies corresponding to the minimum of free energy of the system.

(a)

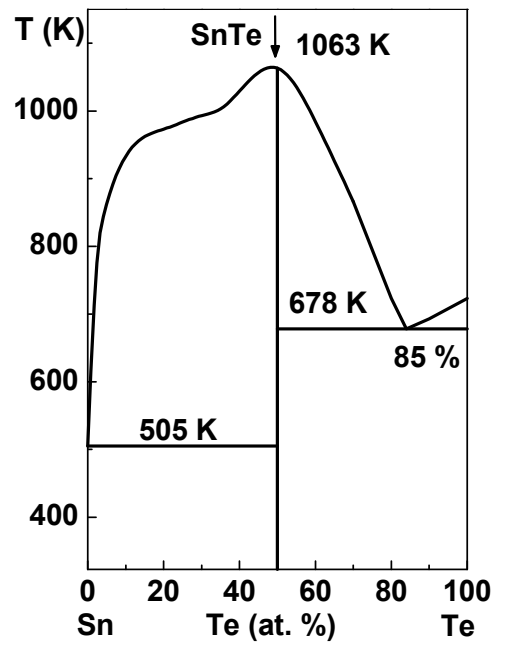

(b)

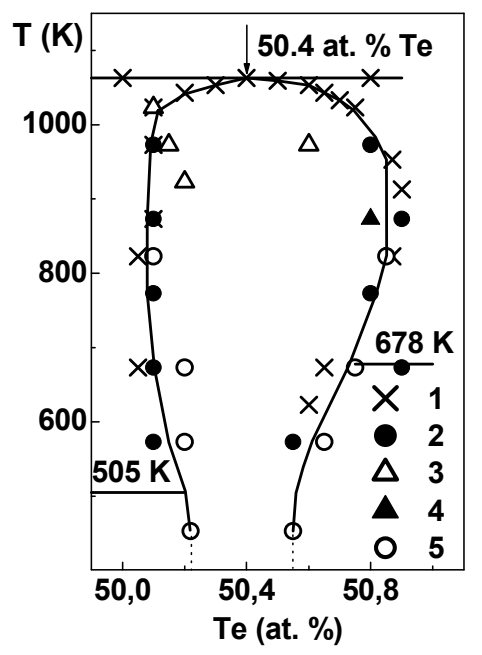

Fig. 1. Sn-Te system phase diagram (a) and homogeneity region of SnTe compound (b). b: 1 - (Brebrick, 1963, 1971; Brebrick \& Strauss, 1964), 2 - (Shelimova \& Abrikosov, 1964), 3 - (Tairov et al., 1970), 4 - (Mazelsky \& Lubell, 1963), 5 - (Rogacheva et al., 1991a).

The influence of defects on mechanical properties of crystals is well known. That is why one can expect that crystal disordering connected with deviation from stoichiometry must noticeably affect such parameters as ultimate strength, Youngs' modulus, microhardness $H$, etc. $\mathrm{H}$ represents a generalized characteristic of resistance to plastic deformation under contact compression (Grigorovich, 1976). The main mechanism of plastic deformation is dislocation movement which is impeded primarily by other dislocations and point defects. Doping usually leads to deformation hardening of the crystal as a result of elastic and electrostatic interactions between dislocations and IA (Suzuki et al., 1991). Thus, one can suggest that under introduction of NSD, $H$ must increase. According to the literature data (Abrikosov \& Shelimova, 1975), $H$ increases linearly with increasing Te content within the SnTe HR. However, our detailed study (Rogacheva et al., 1986,1991a) showed that the $H$-curves exhibit an anomalous behavior in the vicinity of 50.4 at. \% Te (Fig. 2,b-e). 

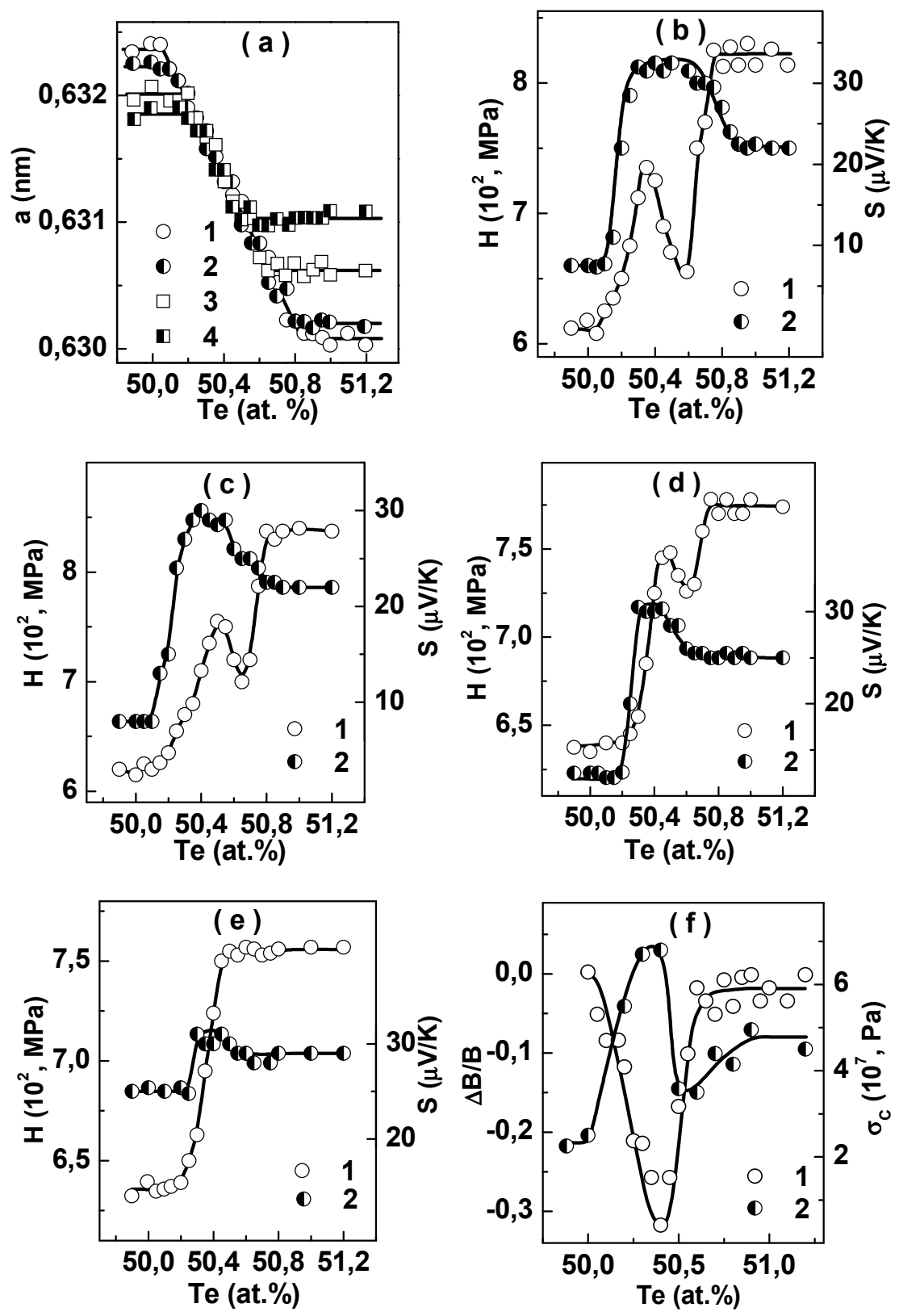

Fig. 2. Concentration dependences of the unit cell parameter $a(\mathrm{a})$, microhardness $H$ (b-e), Seebeck coefficient $S$ (b-e), X-Ray linewidth $\Delta B / B$ (f), and ultimate compressive strength $\sigma_{c}$ (f) in the SnTe homogeneity region after different heat treatments : a: 1 - HT1, 2 - HT2, 3 - HT3, 4- HT4; b-e: 1 - H, 2 - S; b - HT1, c - HT2, d - HT3, e - HT4 (e); f: 1 - $\Delta B / B, 2$ - $\sigma_{c}$ (Rogacheva et al., 1986, 1991a). 
The inflection near 50,4 at.\% Te was also observed in the dependence of ultimate compressive strength on Te content (Fig.2,f). It can be suggested that the behavior of $H$ is determined by the defect interaction which starts to manifest itself at their sufficiently high concentration. This suggestion is additionally supported by the fact that at 50.4 at. \% Te, the minimum of the X-ray line width $\beta$ is observed (Fig. 2,f) (Rogacheva et al., 1986; 2003a). As all the samples were prepared using the same technique, the smallest value of $\beta$ at 50,4 at.\% Te may indicate the maximum degree of structure ordering.

\subsection{Nonstoichiometric vacancies and lattice dynamics}

Defects affect the character of lattice vibrations and can significantly change the phonon spectrum of crystal. Among the properties determined by the crystal lattice dynamics are heat capacity, the Debye temperature $\theta_{\mathrm{D}}$, thermal expansion coefficient $\alpha$, the probability of Mössbauer effect $f^{\prime}$, phonon thermal conductivity $\lambda_{p}$. One can expect that the presence of NS vacancies in the crystal will lead to an increase in the anharmonicity of atom vibrations leading to a growth in $\alpha$, decrease in $\lambda_{p}, \theta_{D}$ and $f^{\prime}$. However, the situation can change under NSD ordering, when defects form a periodic structure and in the harmonic approximation do not scatter phonons. That is why from the dependences of dynamic properties on the vacancy concentration one can indirectly judge about the character of the vacancy distribution in the cation sublattice.

\subsubsection{Phonon spectrum of SnTe}

The Debye approximation assumes a single atom in a unit cell and only acoustic branches of vibrations (Maradudin, 1968). The existence of a $\theta_{D}(T)$ dependence signals about nonapplicability of the Debye approximation. According to (Gul'tyaev \& Petrov, 1951) at sufficiently high temperatures, $\theta_{D}$ is constant, and its value for SnTe $(139 \pm 3 \mathrm{~K})$ is close to the thermodynamic $\theta_{D}(130 \mathrm{~K})$. In (Bukchpan, 1968), $\theta_{D}$ did not change in the range 85-250 K and equaled (132 \pm 3$) \mathrm{K}$. Analysis of the data reported in (Brukhanov et al., 1964; Varnek et al., 1980; Bukchpan, 1968) shows that $\theta_{D}$ determined from $f^{\prime}$ at $290 \mathrm{~K}(132-139 \mathrm{~K})$ practically coincides with the value of $\theta_{D}$ obtained from heat capacity measurements (Gul'tyaev \& Petrov, 1951). Because of the closeness of its vibrational spectrum to the Debye one, SnTe is a convenient object for studying the influence of NS vacancies on the phonon spectrum and dynamic properties of the crystal.

\subsubsection{Nuclear gamma resonance}

The influence of various factors on phonon spectra can be studied by measuring the Mössbauer spectrum parameters $\left(f^{\prime}\right.$, isomer shift $\delta$, linewidth $\Gamma$, absorption area $\left.S\right)$. The introduction of cation vacancies must lead to a disturbance of the phonon spectrum of the crystal (Maradudin, 1968) and, hence, to a change in $f^{\prime}, S$, and $\theta_{D}$. According to (Bekker et al., $1973)$, in the Sn-Te system, the value of $\delta$ practically does not depend on the ratio of constituents, whereas according to (Varnek et al., 1980), $\delta$ changes under increasing nonstoichiometry. In (Bekker et al., 1973), at $300 \mathrm{~K}$ an increase in $\Gamma$ with increasing Te content was observed, while in (Varnek et al., 1980) no additional broadening of lines was registered. According to (Bekker et al., 1973), the area $S$, which is directly proportional to $f^{\prime}$, 
and calculated on its basis values of effective $\theta_{D}$ somewhat increase with increasing degree of deviation from stoichiometry, at the same time in (Varnek et al., 1980; Bekker et al., 1973) it is reported that $\theta_{D}$ does not practically depend on composition. Thus, the data reported by different authors disagree. That is why we carried out a detailed study on the influence of deviation from stoichiometry on the Mössbauer parameters of SnTe (Baltrunas et al., 1986) at $300 \mathrm{~K}$ on bulk samples annealed at $820 \mathrm{~K}$ for 300 hours. A solid solution of $\mathrm{Sn}^{119}$ in $\mathrm{Pd}$ served as $\gamma$-source. The area $S$ was calculated as $S=\pi \cdot f^{\prime} \cdot \Gamma / 2$. The spectra of ${ }^{119}$ Sn atoms represented singlet lines, whose width is typical for highly symmetrical environment of $\mathrm{Sn}$ atoms. The values of $\delta$ were close to the literature data on SnTe (Flinn, 1978) and corresponded to $\mathrm{Sn}^{2+}$. It was found that an increase in Te content is accompanied by a decrease in $\delta$ (Fig. 3,a). The concentration dependences of $\Gamma$ and $S$ are nonmonotonic: in the $\Gamma$ curve, a slight maximum at 50.4 at. \% Te is seen, and in the $S$ curve, there are two maxima: a slight maximum at 50.4 at.\% Te and a distinct one at 50.8 at.\% Te. It is known (Flinn, 1978) that isomeric shift in $\mathrm{Sn}$ is determined by effective numbers of valence $5 \mathrm{~s}$ and $5 \mathrm{p}$ electrons $\left(n_{s}\right.$ and $\left.n_{p}\right): \delta=-0.41+3.10 n_{s}-0.20 n_{s}^{2}-0.17 n_{s} n_{p}$. The chemical bond in SnTe is realized mainly by $p$-electrons with a small contribution of valence $s$-states. The appearance of holes leads to a deficit of valence electrons, which explains the decrease in $\delta$ with increasing hole concentration. The Mössbauer coefficient $f^{\prime}$ is one of the parameters related to $\theta_{D}$ which is determined by the mean-square displacement of atom under thermal vibrations $\left\langle\mathrm{x}^{2}\right\rangle$ :

$$
\mathrm{f}^{\prime}=\exp \left[-\left(4 \pi^{2}\left\langle\mathrm{x}^{2}\right\rangle / \lambda^{2}\right)\right]
$$

and can be calculated using the Debye model (Wertkheim, 1966):

$$
\mathrm{f}^{\prime}=\exp \left[-\frac{\mathrm{E}^{2}}{2 \mathrm{Mc}^{2} \mathrm{k}_{0} \theta_{\mathrm{D}}}\left(\frac{3}{2}+\frac{\pi^{2} \mathrm{~T}^{2}}{\theta_{\mathrm{D}}^{2}}\right)\right]
$$

where $E$ is the transition energy, $M$ is the nucleus mass, $\kappa_{0}$ is the Boltzmann constant. It follows from general considerations that the introduction of defects must result in a local weakening of bonds between particles, a decrease in $f^{\prime}$ and $\theta_{D}$. For example, a decrease in $\theta_{D}$ was registered under introduction of impurities into Ge: for pure Ge, $\theta_{D}=374 \pm 2 \mathrm{~K}$, while for Ge containing $5.410^{19} \mathrm{~cm}^{-3} \mathrm{Ga}, \theta_{D}=362 \pm 2 \mathrm{~K}$ (Zhdanova \& Kontorova, 1965). Growth instead of drop in $\theta_{D}$ under deviation from stoichiometry in the Sn-Te system despite the introduction of charged vacancies can result from the vacancy ordering. The maximum of $S$ at 50.4 at.\% Te supports the idea about the vacancy ordering, showing simultaneously a high sensitivity of the Mössbauer method to the processes of point defect redistribution. The sharp growth in $S$ at 50.75 at.\% Te may be an evidence of the formation of another ordered phase with a higher strength of chemical bonds and higher $\theta_{D}$, and existing in a narrow range of concentrations near the boundary of the SnTe HR. As the distance between vacancies corresponding to 50.8 at. $\%$ Te is $d=2 a$, the ordering process is very likely. The increase in vacancy concentration leads to an increase of the distribution asymmetry of the electric charge around the Mössbauer nucleus and to the broadening of linewidth (Fig. 3,a). The decrease of $\Gamma$ after 50.4 at.\% Te is caused apparently by the redistribution of vacancies which results in their symmetrization around the Mössbauer nuclei. 

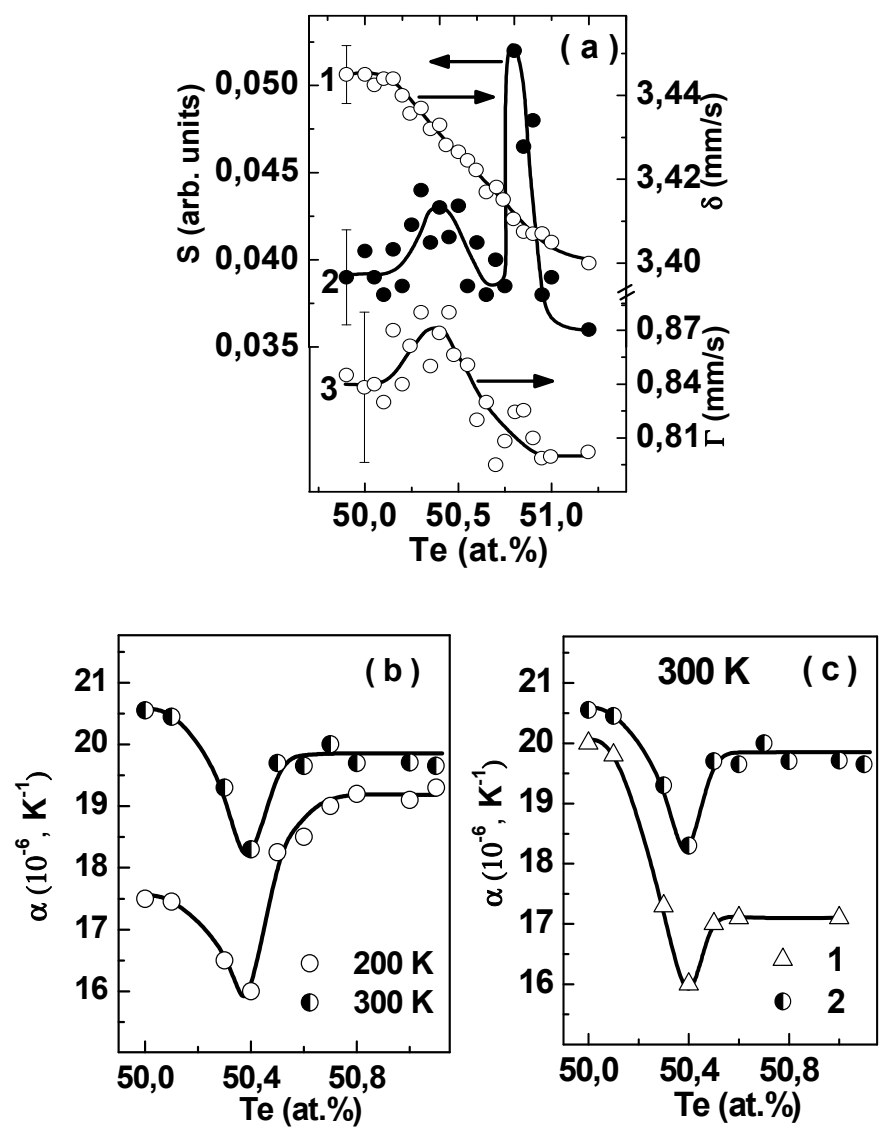

Fig. 3. The isomer shift $\delta$, the area under the Mössbauer spectrum line $S$, the linewidth $\Gamma(\mathrm{a})$, and thermal expansion coefficient $\alpha(b, c)$ vs Te concentration in the Sn-Te system. $\delta$ is recalculated relative to the $\mathrm{Ba}^{119} \mathrm{SnO}_{3}$ source (Baltrunas et al., 1986). a: $1-\delta, 2-S, 3-\Gamma$; b: dynamic regime; c: 1 - stationary regime; 2 - dynamic regime (Nashchekina et al., 2008).

\subsubsection{Phonon thermal conductivity}

Measurement of phonon thermal conductivity $\lambda_{p}$ is one of the tools for studying dynamic irregularity caused by the presence of point defects. Anharmonicity of the lattice vibrations leads to scattering of phonons by phonons, which determines a finite thermal conductivity even in case of undoped crystal. The introduction of point defects results in additional scattering of phonons by point defects and can intensify phonon-phonon scattering due to the increase in cubic anharmonicity of lattice vibration. The authors of (Damon, 1966) who measured the $\lambda_{p}(T)$ dependences of SnTe monocrystalline samples with various $p$ in the range of $100-500 \mathrm{~K}$ concluded that along with the processes of three-phonon scattering, scattering of phonons by vacancies takes place. It was pointed out that this scattering is rather strong and for the most part is caused by fields of deformations created by vacancies. 
The authors of (Erasova \& Kaydanov, 1968) arrived at the conclusion that $\lambda_{p}$ of SnTe is practically independent of the cation vacancies concentration. We conducted a detailed study of $\lambda_{p}$ of SnTe as a function of the cation vacancy concentration on samples with 49,9-51 at.\% Te (Rogacheva et al., 1995). In Fig. 4,a,b the T-dependences of total $\lambda$, electronic $\lambda_{e}$ and phonon $\lambda_{p}$ thermal conductivity and thermal resistance $1 / \lambda_{p}$ in the range of $300-650 \mathrm{~K}$ are presented, and in Fig. 4,c,d , the isotherms of these parameters are given. Analysis of the $1 / \lambda_{p}$ (T) dependences showed that for all samples a linear dependence $1 / \lambda_{p}=\mathrm{AT}+\mathrm{B}$ is observed. The value of $A$ characterizing the slope of the straight lines remains constant, whereas the value of B corresponding to additional thermal resistance caused by vacancies increases with increasing Te content. The straight line drawn through the origin of coordinates parallel to the obtained lines corresponds to the temperature dependence of $1 / \lambda_{p}$ of a hypothetical defectless SnTe. It is known (Berman, 1979; Mogilevskii \& Chudnovskii, 1972; Drabl \& Goldsmit, 1963) that if thermal conductivity is associated only with three-phonon processes, the Eiken law is fulfilled: $1 / \lambda_{p}=k T / \theta_{D}{ }^{3}$, where $k$ is the coefficient taking into account the degree of anharmonicity of crystal lattice vibrations. According to the obtained results, the introduction of vacancies does not lead to breaking the Eiken law, and the linear behavior of the temperature dependences of $1 / \lambda_{p}$ indicates that thermal resistance caused by vacancies does not depend on temperature and is determined only by scattering by defects. This result is in good agreement with the theory: at $T>\theta_{D}$ thermal resistance caused by defects does not depend on temperature. SnTe satisfies these conditions, as $\theta_{D} \cong 130 \mathrm{~K}$. The constancy of the $1 / \lambda_{p}$ straight lines slope shows that the coefficient of proportionality in the Eiken law does not virtually change under increasing degree of deviation from stoichiometry, and this fact is another argument in favor of the suggestion about the vacancy ordering. It follows from Fig. 4 that for the defectless SnTe $\lambda_{p} T=1600 \mathrm{~W} / \mathrm{m}$. Theoretical calculations of this parameter performed in different works yield rather close values: 1620 W/m (Julian C.L., 1965), 1300 W/m (Keyes R.W., 1959, Klemens P.G., 1955). From $1 / \lambda_{p}$ dependences on the IA concentration one can estimate thermal resistance produced by each IA and the cross-section of phonon scattering $\sigma$, using the equation (Ioffe, $1954)$ :

$$
\frac{\lambda}{\lambda_{0}}=1+\frac{\mathrm{N}}{\mathrm{N}_{0}} \cdot \Phi \cdot \frac{1_{0}}{\mathrm{a}}
$$

where $N$ is the IA concentration; $N_{0}$ is the number of atoms in $1 \mathrm{~cm}^{3}$ of the crystal; $a$ is the distance between neighboring atoms; $l_{0}$ is the phonon mean free path; $\Phi$ is the factor in the equation $\sigma=\Phi \mathrm{a}^{2} ; \lambda$ and $\lambda_{0}$ are the $\lambda_{\mathrm{p}}$ of the crystal lattice with and without impurities. In our case, cation vacancies play the role of IA. The mean free path $l_{0}$ is calculated using the Debye equation: $\lambda_{0}=\left(\mathrm{C}_{\mathrm{V}} \cdot l_{0} \cdot \mathrm{V}_{\mathrm{gr}}\right) / 3$, where $\mathrm{C}_{\mathrm{v}}$ is the heat capacity per $1 \mathrm{~cm}^{3}$ and $V_{g r}$ is the mean group velocity $\left(V_{g r}=(E / \rho)^{1 / 2}\right.$, where $E$ is the elastic modulus and $\rho$ is the density). From equation (2) we obtain $\sigma \cong 10 \mathrm{a}^{2}$ and $\Phi=10$. For comparison, values of $\Phi$ under doping of $\mathrm{PbTe}$ with I, Cl, Br are $\Phi=3-3.7$ (Ioffe, 1954). For isovalent IA in PbTe, $\sigma$ is much smaller than for I, Cl, Br (by a factor of $\sim 5$ ). It was established (Drabl \& Goldsmit, 1963) that for I and $\mathrm{Cl}$ impurities in $\mathrm{Bi}_{2} \mathrm{Te}_{3}, \Phi$ is 13 , and the large value of $\Phi$ was attributed to the fact that I and $\mathrm{Cl}$ occupy interstitial sites and strongly scatter phonons. It follows from our data that crosssection of phonon scattering by NS vacancies is sufficiently large. 

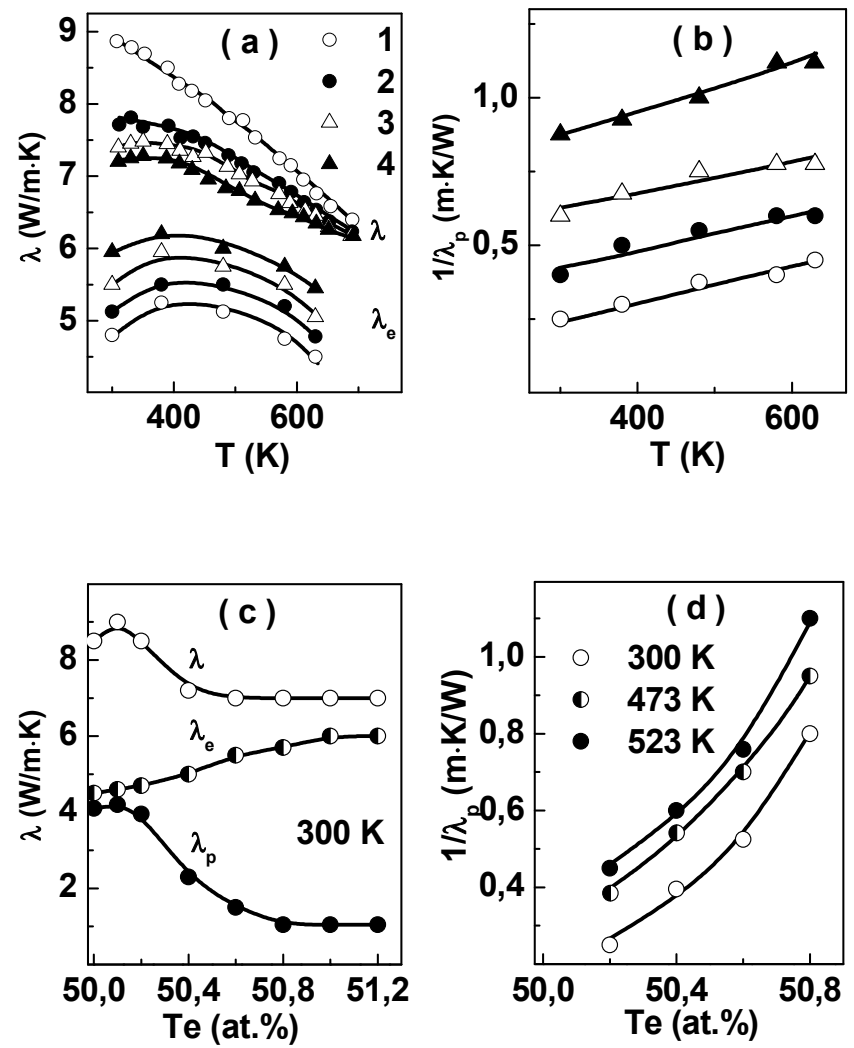

Fig. 4. Temperature $(\mathrm{a}, \mathrm{b})$ and concentration $(\mathrm{c}, \mathrm{d})$ dependences of total $\lambda(\mathrm{a}, \mathrm{c})$, electronic $\lambda_{e}$ $(\mathrm{a}, \mathrm{c})$ and phonon $(\mathrm{c})$ thermal conductivities and thermal resistance $1 / \lambda_{\mathrm{P}}(\mathrm{b}, \mathrm{d})$ of SnTe. a,b: 1 50.2 at. \% Te, 2 - 50.4 at. \% Te, 3 - 50.6 at.\% Te , $4-50.8$ at. \% Te (Rogacheva et. al, 1995).

In accordance with the Klemens theory (Klemens, 1955) scattering of phonons by defects can be described taking into account contributions of three factors: local change of mass $\Delta M / M$, strength of atomic bonding $\Delta G / G$, elastic distortion of the crystal caused by the difference in effective sizes of the atom (ion) and the substituting defect $\Delta R / R$. The total scattering crosssection according to Klemens is as follows

$$
\sigma=\frac{\mathrm{V}_{0}^{2} \omega^{4}}{4 \pi \mathrm{V}^{4}}\left\{\left(\frac{\Delta \mathrm{M}}{\mathrm{M}}\right)^{2}+2\left[\frac{\Delta \mathrm{G}}{\mathrm{G}}-\frac{\mathrm{b}}{\sqrt{5}} \cdot \gamma \cdot \frac{\Delta \mathrm{R}}{\mathrm{R}}\right]^{2}\right\}
$$

where $V_{0}$ is the volume per atom, $\Delta G$ is the local change in the elastic modulus, $\Delta M$ is the local change of mass, $\gamma$ is the Gruneisen parameter, $b=14.2$ (Berman, 1979). Assuming $\omega=\omega_{\max }=k_{0} \theta_{D} / h, \theta_{D}=130 \mathrm{~K}, \gamma=2, \Delta R / R=0.13, V_{0}=3110^{-30} \mathrm{~m}^{3}$, we obtain $\sigma \cong 10 \mathrm{a}^{2}$, which practically coincides with $\sigma$ determined on the basis of the experimental data. 


\subsubsection{Coefficient of thermal expansion}

Change in the crystal volume under changing temperature is caused by an asymmetry of the resulting interaction potential in the crystal lattice, manifesting itself as the interaction of vibrations of the phonon spectrum. The degree of the volume change is characterized by the coefficient of volume expansion $\beta$ (Novikova, 1974):

$$
\beta=\frac{1}{\mathrm{~V}}\left(\frac{\partial \mathrm{V}}{\partial \mathrm{T}}\right)_{\mathrm{P}}=\frac{1}{\mathrm{~V}}\left(\frac{\partial^{2} \mathrm{~F}}{\partial \mathrm{P} \cdot \partial \mathrm{T}}\right), \beta \cong 3 \alpha,
$$

where $F$ is the free energy, $\alpha$ is the coefficient of linear expansion. As $F$ is an additive function, and can be considered for solids as the sum of free energies of the crystal lattice, electron gas, magnons and so on, $\beta$ is also an additive function: $\beta=\beta_{L}+\beta_{l}+\beta_{m}+$, where $L, l$, $m$ denote lattice, electronic and magnetic contributions respectively. As a rule, at $T>0.1 \theta_{D}$ the lattice contribution plays the main role. It is known that the introduction of IA usually leads to an increase in $\alpha$ of the crystal (Novikova, 1974; Zhdanova \& Kontorova, 1963; Abrikosov et.al., 1981, 1982). This is in good agreement with the theoretical calculations performed for diluted solid solutions (Varisov et.al, 1966; Timmesfeld \& Elliot, 1970) according to which the increase in $\alpha$ is connected with a growth in configurational entropy of the ideal crystal under introduction of IA as well as with the effect of free charge carriers on heat capacity and the Gruneisen parameter. In accordance with (Timmesfeld \& Elliot, 1970), the change in $\alpha$ under introduction of substitutional point defects occurs due to: i) a change in elastic constants in the vicinity of the defect, ii) a change in anharmonicity near the defect, iii) a change in the normal model of a crystal with defects. These three factors can partially compensate each other, so the resulting change in $\alpha$ can be positive or negative. In (Masharov, 1963), a dynamic model of the crystal with a small concentration of vacancies or IA is suggested. According to this model, "defect" crystal can be considered as an ideal one with the phonon energy depending on the defect concentration. Using this model, the authors of (Varisov et al., 1966) calculated corrections in $\alpha$ caused by vacancies and associated with the change in configurational potential energy.

The effect of deviation from stoichiometry on thermal expansion of phases of variable composition has not been investigated in detail (Novikova \& Shelimova, 1965,1967; Belson \& Houston, 1970). That is why we have conducted studies on the influence of deviation from stoichiometry, temperature, and kinetic factors on $\alpha$ of SnTe crystals (Rogacheva et al., 1993; Rogacheva, 2003b; Nashchekina et al., 2008). The $\alpha(T)$ dependences in the range 5-300 $\mathrm{K}$ or $77-300 \mathrm{~K}$ were obtained under two regimes. The stationary regime consisted in cooling samples down to the liquid helium temperature slowly and then heating up to room temperature, keeping at the measurement temperatures until complete temperature stabilization is reached. Under the dynamic regime samples were quickly quenched in liquid nitrogen, and the $\alpha(T)$ curves were measured under subsequent rapid heating. The first regime was close to quasi-equilibrium conditions, whereas the other one corresponded to apparently non-equilibrium conditions of heating. For both regimes the minimum value of $\alpha$ within the SnTe HR was observed at 50.4 at.\% Te (Fig. 3, b,c). It can 
be suggested that an increase in the dynamic stability of the SnTe crystal lattice under increasing concentration of NSD is connected with ordering of vacancies. It is known that $\alpha=\beta \cdot k_{0} / \mathcal{E}^{2} \cdot a$, where $\varepsilon$ is the coefficient of quasi-elastic bond, $\beta$ is the coefficient of anharmonicity, $a$ is the distance between atoms; $k_{0}$ is the Boltzmann constant. Although the equation is rather rough and corresponds to a two-atom model, under the condition of preservation of the crystal structure, on the basis of $\alpha$ values one can make comparative estimates of the degree of crystal anharmonicity and values of average displacements of atoms from the equilibrium positions: $x=\alpha \cdot a \cdot T$ - proportional to temperature. The calculation shows that the value of $x$ for SnTe is $\sim 0,005 a$ at $300 \mathrm{~K}$. In comparison with $\mathrm{SnTe}$, in $\mathrm{Sn}_{0,984} \mathrm{Te}$ the number of vacancies in the cation sublattice increases from 0,4 to 1,6 $\%$, and $\alpha, \beta / \mathcal{E}^{2}, x$ decrease by $\sim 6 \%$. There is a simple relationship between $\alpha$ and $\theta_{D}$ (Novikova, 1974): $\theta_{\mathrm{D}}=\sqrt{\mathrm{A} /\left(\alpha \mathrm{V}^{2 / 3} \mathrm{M}\right)}$, where $M$ is the molecular weight; $A$ is the constant depending on the type of chemical bond. The estimate shows that as $\alpha$ changes by $\sim 6 \%$, $\theta_{D}$ increases by $\sim 5 \%$. The calculation of the change in $\theta_{D}$ within the SnTe HR performed on the basis of the Mössbauer data in (Bekker, 1973) demonstrated that for $300 \mathrm{~K}$ the change in $\theta_{D}$ amounts to $\sim 8 \%$, which is in good agreement with the result of our calculation of the change in $\theta_{D}$ based on the data on $\alpha$.

\subsection{Electronic properties and band structure}

\subsubsection{Electronic properties}

Deviation from stoichiometry in semiconductor compounds leads to perturbation not only in the crystal lattice but also in the electronic subsystem of crystal. In Figs. 5,6 the temperature and concentration dependences of $S, \sigma, R_{H}, p$, Hall mobility $\mu_{H}$, and NernstEttinsgausen coefficient $Q_{\perp}$ for SnTe samples with different degrees of deviation from stoichiometry are presented (Laptev \& Rogacheva, 1986, 1988). With increasing Te content, $\sigma$ and $p$ increases, $\mu_{H}$ decreases. The $S$ - composition dependence has a maximum at $\sim 50.4$ at.\% (Fig. 2,b-e), and this fact is usually explained on the basis of the model of the valence band which consists of two subbands with different densities of states. From the concentration dependences of $S, \sigma, R_{H}$ and $\mathrm{Q}_{\perp}$ in a weak magnetic field assuming single type of carriers, isotropic case, strong degeneration, elastic scattering, and absence of phonon drag, we estimated scattering parameter $t$ and effective mass of density of states at the Fermi level $m_{1}^{*}$ (Fig. 6). The calculations showed that $t=0$, which corresponds to scattering by close-range components of vacancy potential in the low temperature range and to acoustic scattering at high temperatures. The $m_{1}^{*}(p)$ dependence is described by the equation: $\mathrm{m}^{*}{ }_{1}=0.28 \mathrm{~m}_{0}\left(\mathrm{p} / \mathrm{p}_{\mathrm{O}}\right)^{2 / 3}$, where $p_{0}$ is the minimum $p$ in the SnTe HR. Such dependence indicates band spectra nonparabolicity, which does not correspond to the dispersion laws (Kane, 1957) or (Cohen, 1961). The T- dependences of kinetic coefficients are characterized by maxima in the $R_{H}(T)$ and $S(T)$ curves, a drop in $R_{H}(T)$ and $S(T)$ at high temperatures, a sharp growth in $S$ at $T \sim 300-500 \mathrm{~K}$ for the samples with a low $p$. The attempts to explain the anomalies using existing models (Andreev, 1967; Efimova et al., 1965; Kaydanov et al., 1967), i.e. by the increasing contribution of intrinsic conductivity to kinetic effects at $\mathrm{T}>600 \mathrm{~K}$ fail, because within those models the temperature of maxima in the $R_{H}(T)$ and $S(T)$ curves is estimated at more than $1000 \mathrm{~K}$. 

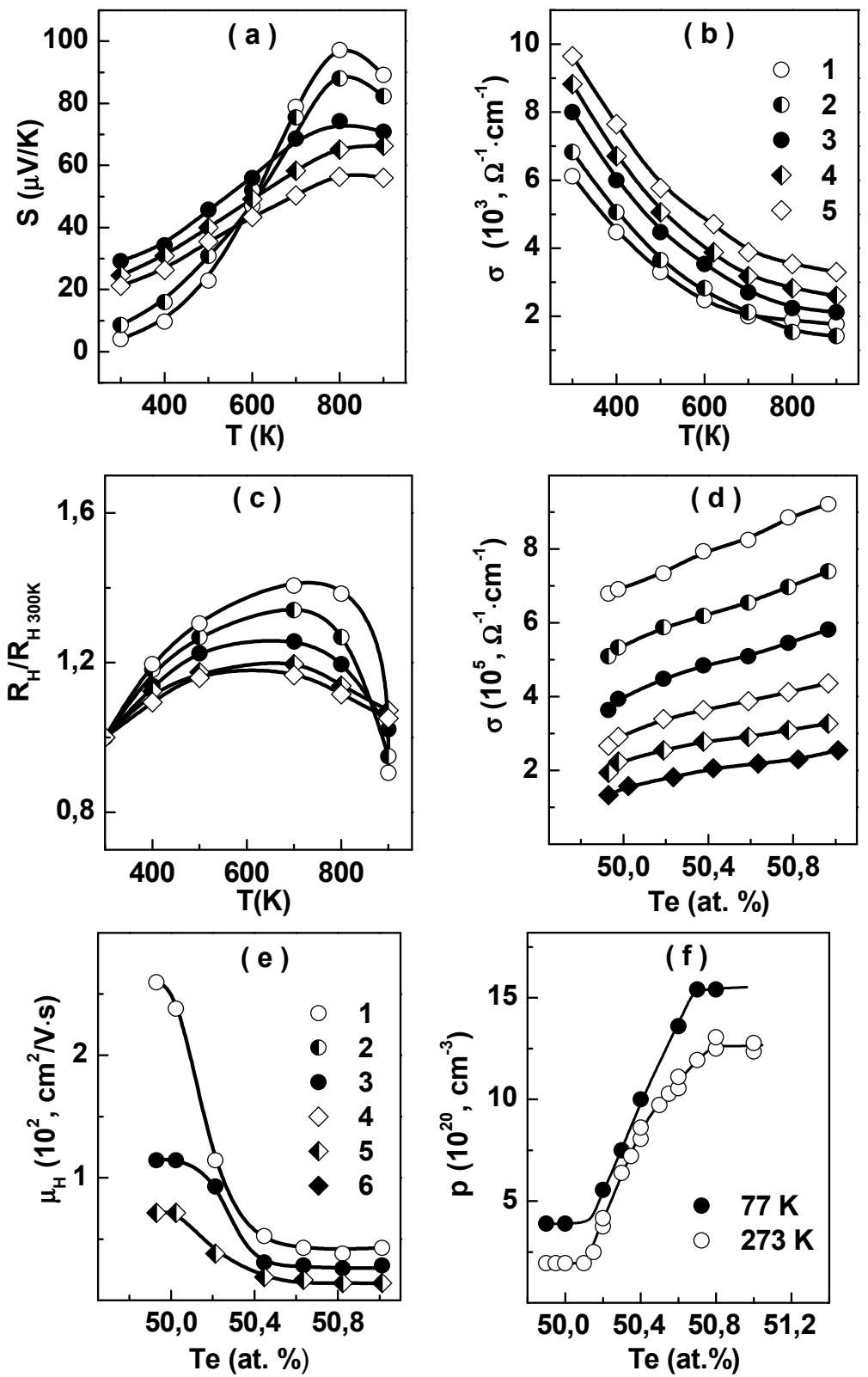

Fig. 5. Temperature (a-c) and concentration (d-f) dependences of the Seebeck coefficient $S$ (a), electrical conductivity (b, d), Hall coefficient (c), Hall charge carrier mobility $\mu_{\mathrm{H}}(\mathrm{e})$, and hole concentration p (f). a,b,c: $1-50.0$ at. \% Te, $2-50.2$ at. \% Te, $3-50.4$ at. \% Te, $4-50.6$ at.\% Te, 5 - 50.8 at. \% Te; d, e, f: 1 - 273 K, 2 - 373 K, 3 - 473 K, 5 - 573 K, 6 - 773 K (Laptev \&Rogacheva, 1986, 1988). 

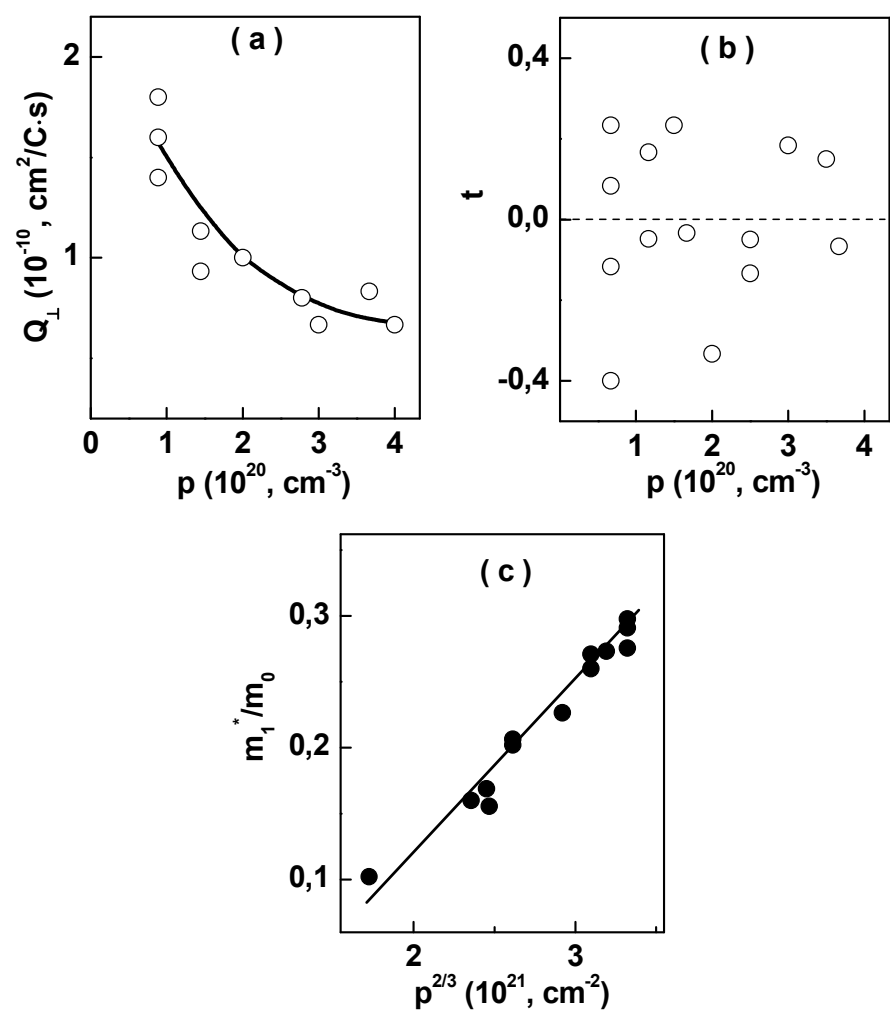

Fig. 6. Dependences of the transverse Nernst-Ettinsgausen coefficient $Q_{\perp}$ (a), scattering parameter $t$, and effective mass of the density of states $m_{1}{ }^{*} / m_{0}$ (c) on the hole concentration $p$ in SnTe (Laptev \&Rogacheva, 1986, 1988).

\subsubsection{Hall-factor in SnTe}

The number of carriers supplied by each Sn vacancy to the SnTe valence band is of fundamental importance in studies of the energy structure of this compound. It is related to the value of the Hall factor $r$ defined by the relationship $p=r / R_{H} \cdot e$.

Assuming $r=1$, the authors of (Brebrick, 1963) conclude that each Sn vacancy is a doubly ionized acceptor and supplies two carriers. According to (Houston et al., 1963), this conclusion is correct if one assumes that $r=0.6$. For determining $r$ we measured the dependences of longitudinal $\rho_{\mathrm{zz}}$ and transverse $\rho_{\mathrm{xx}}$ magnetoresistance and $R_{H}$ on magnetic field $H$ for SnTe samples with $p=(0.75-2.5) \cdot 10^{26} \mathrm{~m}^{-3}$ (Kolomoets et al., 1986) (Fig. 7,a,b). All the dependences exhibited oscillations whose amplitude increased with increasing $p$ at constant $H$ (Fig. 7,b.). It was found that $r=0.9-1.0$. Comparison of this value of $r$ with the dependence of $p$ on the concentration of Te in the Sn-Te system (Fig. 7,c) led to the conclusion that each vacancy supplies four holes to the valence band. This conclusion was used to forecast the effect of vacancies on the energy spectrum of SnTe (Laptev \& Rogacheva, 1986, 1988). 

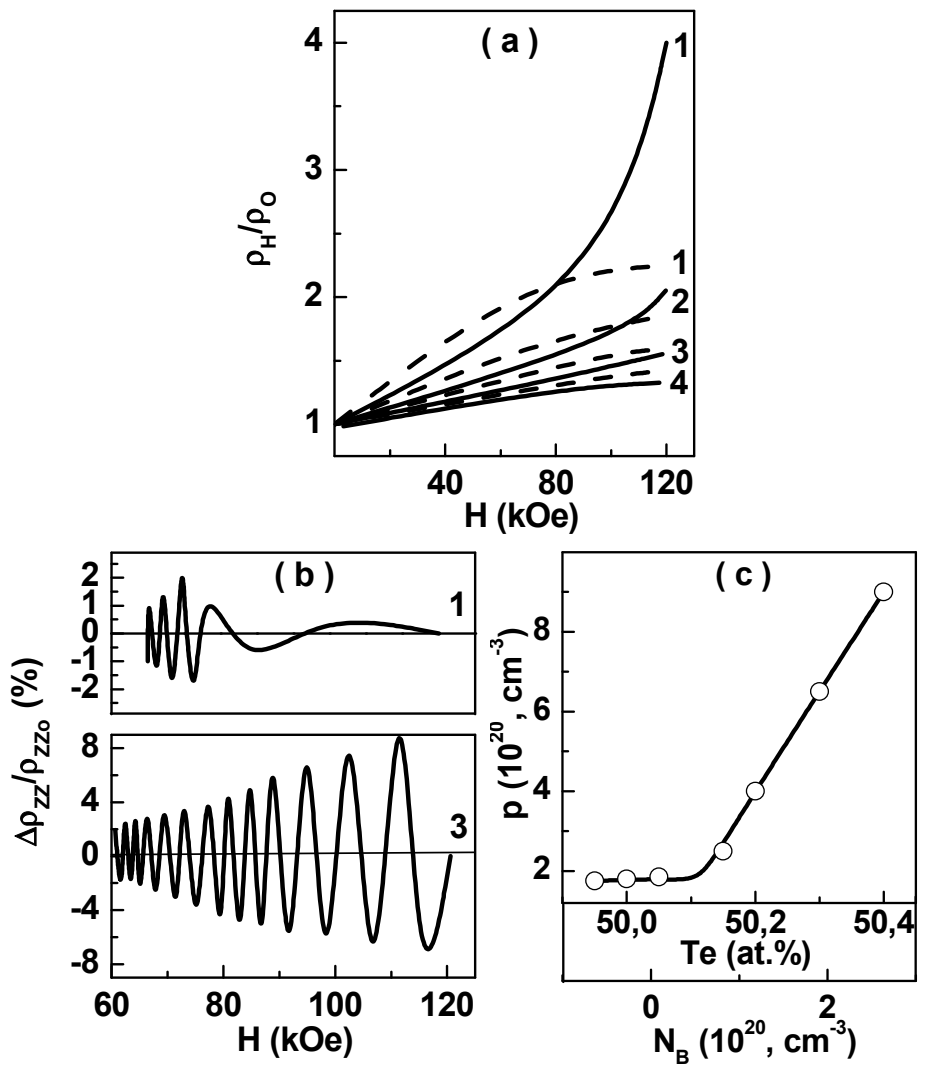

Fig. 7. Dependences of the longitudinal $\rho_{x x}$ magnetoresistance on the magnetic field: a) monotonic part; b) oscillatory part. The dashed curves represent $\rho_{\mathrm{ZZ}}$. c) dependence of the hole concentration $p$ in SnTe on the Te content and on the concentration of cation vacancies (Kolomoets et.al, 1986).

\subsubsection{New model of the valence band}

The transport properties of SnTe exhibit anomalies which are common for all IV-VI compounds: a sharp change of $R_{H}$ under increasing temperature, nonmonotonic character of the $S(p)$ dependence, anomalous concentration and temperature dependences of hole mobility and so on. On the basis of measurements of $R_{H}$ and $S$ in the range of 4,2-300 K the authors of (Allgaier \& Sheie, 1961) suggested a model of two valence bands for SnTe, which was further developed in (Brebrick et al.,1962; Sagar \& Miller, 1962; Brebrick, 1963; Brebrick \& Strauss, 1963; Kafalas et al.,1964; Andreev, 1967; Kaydanov et al., 1967; Rogers, 1968; Rabii, 1969). Analyzing the literature data on the SnTe band structure one can note two circumstances. On the one hand, none of the models describes all experimental results using a single set of parameters of the energy spectrum and hole scattering, nor can explain a number of the facts, in particular, the character of non-parabolicity of the main band extremum, the origin of "heavy" extremum, the concentration and temperature 
dependences of the transport properties and so on. On the other hand, none of the models takes into account high concentration of NSD, although it must significantly affect the electronic spectrum. In (Nimtz G., Schlicht, 1985; Heinrich, 1979; Kaydanov \& Ravich, 1985; Sobolev, 1981) no impurity levels or bands in the SnTe energy gap were detected. According to the theoretical estimations (Kaydanov \& Ravich, 1985), the defect potential in narrow-gap semiconductors like SnTe must be short-range due to interband screening, significant for a small energy gap, and a large value of static dielectric constant connected with the presence of soft modes. Highly localized potential must result in the formation of deep levels, the impurity state energy can fall within a zone of allowed energies creating a resonant (quasi-local) state. Disturbing potential of vacancies is one of the highest among defects. If a vacancy is not charged, this potential is highly localized and rapidly decreases in comparison with the potential created by a donor or acceptor impurity (Parada \& Pratt, 1969).

We proposed a model of the SnTe energy band structure taking into account a high level of self-doping in this compound (Laptev \& Rogacheva, 1986, 1988), which allowed us to interpret the temperature and concentration dependences of transport properties satisfactorily. According to the model, in the valence band, a resonant band of deep vacancy levels playing the role of additional "heavy" extremum, is formed (Fig.8, a). The band width, number of states and carrier dispersion within the resonant band depend on the vacancy concentration. For description of conductivity over the resonant band, a model of narrow impurity band was applied using approximation of strong coupling. We made assumption about an ordered distribution of vacancies, which was supported by the results of studies of the properties within the SnTe HR. Comparing the results of the theoretical calculations of the concentration and temperature dependences of the kinetic coefficients based on the proposed model with the experimental data we determined a set of parameters for SnTe. At $300 \mathrm{~K}, \Delta \mathrm{E}_{\mathrm{g}}=0.2 \mathrm{eV}$; the distance from the edge of the valence band to the vacancy band is equal to $\Delta \mathrm{E}_{\mathrm{V}}=0.42 \mathrm{eV}$ and does not depend on temperature. Within the $\mathrm{HR}$, the vacancy band width changes from 0,038 to $0,19 \mathrm{eV}$, and the effective mass - from 2,08 to $1,53 \mathrm{~m}_{0}$. This model lends itself for the interpretation of a wide range of phenomena, not enough satisfactorily explained by the other models of the SnTe band structure.
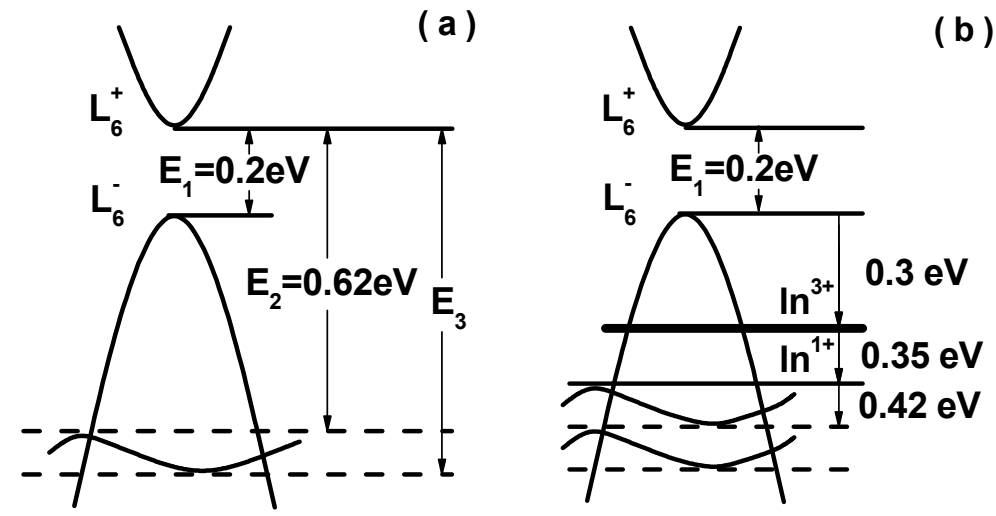

Fig. 8. Models of the band structures of SnTe (Laptev \&Rogacheva, 1986, 1988) (a) and SnTe doped with In (Rogacheva \& Laptev 1999) (b). 
It follows from the said above that when studying the energy spectrum of heavily NS semiconductor phases, it is necessary to take into consideration the possibility of the formation of "defect" bands. The specificity of the "defect" bands consists in the dependence of its parameters on the concentration of defects. The proposed method determines a new approach to study of the band structure of IV-VI compounds, which can be extended to other phases.

\subsection{Discussion}

It is seen from the results described in Section 2 that in the SnTe HR, the concentration dependences of different physical properties, which characterize both the electronic and lattice subsystems of the crystal, exhibit nonmonotonic behavior: practically in all dependences there are peculiarities (extrema, inflection points) in the vicinity of the composition 50.4 at.\% Te corresponding to the maximum in the melting curves.

As was mentioned above, point defects can be considered as noninteracting structural elements only at sufficiently small concentrations. Under increasing concentration, their interaction stimulates the processes of structure ordering. According to the recent theoretical studies (Mycielski, 1986), the Coulomb interaction between impurity centers leads to a uniform distribution of impurity and the formation of superstructure of ionized defects in semiconductors. At present, it is well known that in case of strongly NS phases, wide HR often split into a number of ordered phases with a more restricted nonstoichiometry (Collongues,1974, 1993). Analysis of possible variants of ordered distribution of NS cation Sn vacancies at different their concentrations showed (Rogacheva et al., 1986,1991a) that in the range of the SnTe HR the following types of ordering are theoretically feasible: 1) the location of vacancies in the sites of a primitive unit cell with a parameter $a=4 a_{0}$, where $a_{0}$ is the unit cell parameter of SnTe with random distribution of vacancies, the average distance between vacancies $d$ corresponding to $d=a=4 a_{0}$. This variant of ordering can be realized at 50.1 at.\% Te near the boundary of the HR on the Sn side; 2) the location of vacancies in the sites of a fcc lattice with $a=4 a_{0}, d=2.8 a_{0}$ can occur at 50.4 at.\% Te; 3) the distribution of vacancies over the sites of a primitive unit cell with a unit cell parameter $a=2 a_{0}$ and $d=2 a_{0}$ can take place at 50.8 at.\% Te, i.e. near the HR boundary on the Te side. The results of the analysis allows us to suggest that the maximum within the SnTe HR thermal stability of the sample containing 50.4 at.\% Te is connected with the formation of a fcc lattice of vacancies and leads to the appearance of the anomalies in the concentration dependences of the properties. The results of the Mössbauer studies (see 2.3.2) indicate the possibility of the formation of another ordered phase at 50.8 at. \% Te.

Earlier in a large number of solid solutions we observed concentration anomalies of the properties in the range of small impurity concentrations ( 1 at.\%) (Rogacheva, 1989, 2003; Rogacheva \& Krivulkin, 2001, 2002; Rogacheva et.al., 1998) and suggested that there exists a phase transition from diluted to concentrated solid solutions connected with the formation of percolation channels (Rogacheva, 1993). Reaching the percolation threshold like any critical phenomenon usually manifests itself through the appearance of anomalies in the concentration dependences of properties. If one treats NSD as IA, then again there must exist a critical concentration corresponding to the transition from the defect discontinuum to defect continuum. In terms of this assumption the peculiarities of properties in the vicinity 
of 50,4 at. \% Te can be attributed to reaching the percolation threshold in the vacancy subsystem, when the process of vacancy interaction spreads over the entire system stimulating long- or short-range ordering. Considering the observed critical phenomenon in the framework of the percolation theory (Stauffer \& Aharony, 1992) and assuming that peculiarities in the concentration dependences of properties within HR correspond to the percolation threshold, it is possible to calculate radius of the NSD "action sphere" $R_{o}$ from the composition, at which peculiarities are observed, using the equation $4 / 3 \pi N_{c}\left(2 R_{o}\right)^{3} \approx 2.7$, where $N_{c}$ is the average number of sphere centers per volume unit. The calculation shows that for SnTe $R_{\mathrm{o}} \approx 1.4 a_{0}$, where $a_{0}$ is the unit cell parameter. The formation of continuous chains of NSD upon reaching the percolation threshold can stimulate such redistribution of NSD in the crystal lattice, which would lead to the realization of their configuration corresponding to a minimum of the thermodynamic potential. The fact that at 50.4 at. $\% \mathrm{Te}$, we observed minima of the $\Delta B / B, \alpha$, and a maximum of $f^{\prime}$ is an evidence in favor of NSD (vacancies) ordering in SnTe.

\section{Temperature phase transitions in SnTe}

The existence of the peculiarities in the concentration dependences of the properties within the SnTe HR indicates the presence of phase transitions (PT) connected with the defect redistribution, whose character must depend on composition and temperature. That is why we carried out a complex of works related to detecting possible temperature PT associated with the processes of NSD self-organization. It was mentioned above that SnTe undergoes a ferroelectric phase transition (FPT) at a temperature $T_{c}$ close to $100 \mathrm{~K}$. It is known that $T_{C}$ decreases with increasing hole concentration $p$, and FPT is not observed for $p_{77}=(7-10) 1026 \mathrm{~m}^{-}$ 3, i.e. starting from the composition $\sim 50.4$ at.\% Te (Kobayashi et al., 1976; Sugai et al., 1977; Brilson et al, 1974; Iizumi et al., 1975). The latter circumstance explains the significant discrepancy in $T_{c}$ values reported in different works. Some authors (Fano et al., 1977; Bashkirov et al., 1985; Grassie et al., 1979) reported a much higher value of $T_{C}(160 \mathrm{~K}$ and $140-145 \mathrm{~K}$ ) and presumed the existence of at least two PT in SnTe. The structural instability of SnTe is usually attributed to the softening of the transverse optical mode with zero momentum as a result of the interband electron-phonon interaction (Littlewood,1982; Suski, 1985). The authors of (Khandozhko et al.) observed steps at $T_{n}=376 / n(n=1-6)$ in the $T$ dependences of resonant fields of ${ }^{119}$ Sn nuclei in NMR experiments and suggested that the registered effects are associated with the movement of the crystal lattice defects under a change in temperature.

\subsection{Ferroelectric phase transition}

For studying the structural instability of a ferroelectric type in SnTe crystals and thin films, we used dilatometry, diffractometry, and measurements of transport properties (Rogacheva et al., 1993; Nashchekina et.al., 1995, 1998, 1999a,1999b, 2008). The studies were conducted on SnTe samples with 50.0-50.8 at.\% Te. For the stoichiometric SnTe, in the $\alpha(T)$ curve near 90-100 K (Rogacheva et al., 1993; Nashchekina et.al., 1999b), a distinct $\lambda$-anomaly typical of the second-order PT (Fig. 9,a) was observed. In the $a(T)$ dependence in the range 90-100 K (Fig. 9,b), there was also registered an anomalous section (Nashchekina et.al., 1999c). In Fig. 
10, a,b,c, the $\sigma(T,) R_{H}(T)$, and $\mu_{H}(T)$ dependences are shown (Nashchekina et.al., 1999b). For all samples, $\sigma$ decreases with growing temperature, and for the stoichiometric sample, in the $\sigma(T)$ curve in the vicinity of $90-95 \mathrm{~K}$ there is observed an anomaly manifesting itself through additional drop in $\sigma$. In the samples with 50.4 and 50.6 at.\% Te no anomalies are detected. As temperature increases up to $100-150 \mathrm{~K}, R_{H}$ practically does not change (which is characteristic of semiconductors with a high degree of degeneration), but then $R_{H}$ grows monotonically, which is usually interpreted in terms of the two-band model of the valence band (Nimtz \& Schlicht, 1985; Ravich et al,1970). No temperature anomalies of $R_{H}$ were observed in the vicinity of the FPT for all bulk samples investigated. This indicates that FPT in SnTe is not accompanied by any noticeable change in $p$, but additional scattering of carriers takes place leading to additional decrease in mobility $\Delta \mu_{H}$. On the basis of the experimental data, $\Delta \mu_{H}$ was calculated. Its maximum value corresponds to $T_{C}=93 \mathrm{~K}$ and reaches $\sim 8 \%$ (see the insert of Fig. 10,c). Similarly, additional electrical resistance $\Delta \rho$ as a result of FPT was estimated and compared with the results reported in (Kobayashi et al., 1975) and (Grassie et al., 1979), where the $\rho(T)$ dependences were studied for SnTe single crystals with $p_{H 77}=1.2 \times 10^{20} \mathrm{~cm}^{-3}$ and polycrystalline films with $p_{H 77}=0.76 \times 10^{26} \mathrm{~m}^{-3}$, respectively. In Fig. 10,d, the $\Delta \rho(T)$ dependences obtained in (Nashchekina et al., 1999a) and (Kobayashi et al., 1975) are given. The additional enhancement in $\rho$ at $T_{C}$ amounted to $\sim 2 \%$ in (Kobayashi et al., 1975), 8 \% in (Nashchekina et al., 1999a), and 14 \% in (Grassie et al., 1979). The $\Delta \rho$ value increases as we move from single crystals (Kobayashi et al., 1975) to polycrystals (Nashchekina et al., 1999a), and then to polycrystalline films (Grassie et al., 1979). It can be suggested that imperfection of structure induces additional mechanisms of electron-phonon interaction leading to $\Delta \rho$ increase. The growth in $\rho$ near the FPT was explained quantitatively by two alternative mechanisms. The authors of (Kobayashi et al., 1975; Katayama et al., 1976).), who attributed the anomalous behavior of $\rho$ in the range of the FPT to interband scattering of holes by transverse optical phonons near $L$-point of the Brillouin zone, obtained the following equation for $\Delta \rho$ :

$$
\Delta \rho=\mathrm{T} / \gamma\left(\mathrm{T}-\mathrm{T}_{\mathrm{C}}\right) \quad \text { at } \mathrm{T}>\mathrm{T}_{\mathrm{C}} \quad \Delta \rho=\mathrm{T} / 2 \gamma\left(\mathrm{T}-\mathrm{T}_{\mathrm{C}}\right) \text { at } \mathrm{T}<\mathrm{T}_{\mathrm{C}}
$$

where $\gamma$ is the coefficient independent of temperature. For the explanation of the anomaly in $\rho$, the authors of (Minemura \& Morita, 1978) used the model of band-to-band electronphonon interaction, assuming that the interband interaction through deformation potential is weak. In this model, $\Delta \rho$ changes with temperature according to the law: $\Delta \rho \sim \mathrm{T}^{2}$. In Fig. 10,e the dependence of $\Delta \rho$ on $T /\left(T-T_{C}\right)$, where $T_{C}=93 \mathrm{~K}$, is presented. One can notice that there is a good agreement between the experimental data and theoretical predictions (Kobayashi et al., 1975; Katayama et al., 1976).): the slope of the straight line at $\mathrm{T}<<\mathrm{T}_{\mathrm{C}}$ is almost twice as large as that at $\mathrm{T}>\mathrm{T}_{\mathrm{C}}$; in the temperature range near the FPT, there is a significant deviation from the theoretical dependence. Studying the $\sigma(T)$ dependences of the thin films showed that for the SnTe films with $p_{H}=(1.0-2.5) \cdot 10^{26} \mathrm{~m}^{-3}$ near $100 \mathrm{~K}$ in the $\sigma(T)$ curves there is an anomaly associated with the FPT, although less pronounced than for bulk samples. At higher values of $p_{H}$, the anomaly is not detected (Nashchekina et al., 1998). Thus, the results of our studies convincingly confirm the existence of the FPT in the stoichiometric SnTe, the determined value of $\mathrm{T}_{\mathrm{C}}$ being in good agreement with the results of other authors. 

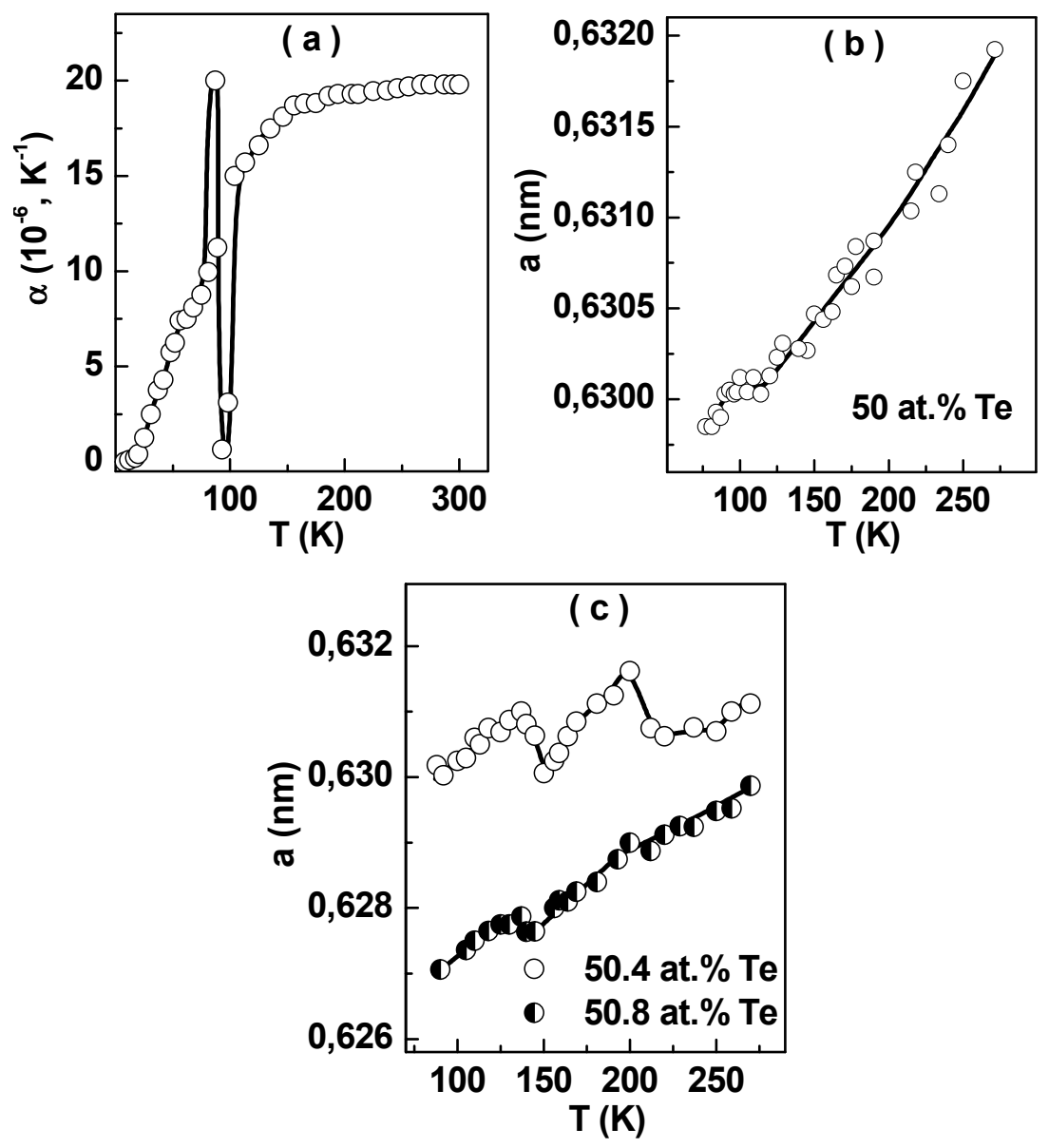

Fig. 9. Temperature dependences of the coefficient of linear expansion for stoichiometric SnTe (a) and unit cell parameter for stoichiometric (b) and nonstoichiometric (c) SnTe

\subsection{Other phase transitions in SnTe}

\subsubsection{X-Ray diffraction studies}

In (Nashchekina et al., 1999b), SnTe with various degrees of deviation from stoichiometry (50.0, 50.4, and 50.8 at.\% Te) was studied by using XRD analysis, and the $a(T)$ dependences were measured during heating in the temperature range 80-290 $\mathrm{K}$ (Fig 9,b,c). It has been mentioned above that the $a(T)$ curve for the sample of the stoichiometric composition exhibits the anomaly in the range $90-100 \mathrm{~K}$ (Fig. 9a), which is apparently associated with the known FPT. For 50.4 at.\% Te, well-pronounced jumps in the unit cell parameter $(\Delta a / a \cong$ $0.015)$ are observed in the intervals $135-150 \mathrm{~K}$ and $200-215 \mathrm{~K}$ corresponding to a negative $a$. At 50.8 at.\% Te these effects become less pronounced (Fig.9,c). The presence of the anomalies in the $a(T)$ curves indicates the instability of the crystal lattice in certain temperature 
intervals. Taking into consideration what was said above regarding the composition of 50.4 at.\% Te (see 2.5), it is natural to assume that the emergence of new PT in SnTe can be due to a change in the equilibrium conditions and the type of polymorphism in the presence of vacancies as well as due to redistribution of intrinsic defects as a result of their interaction.
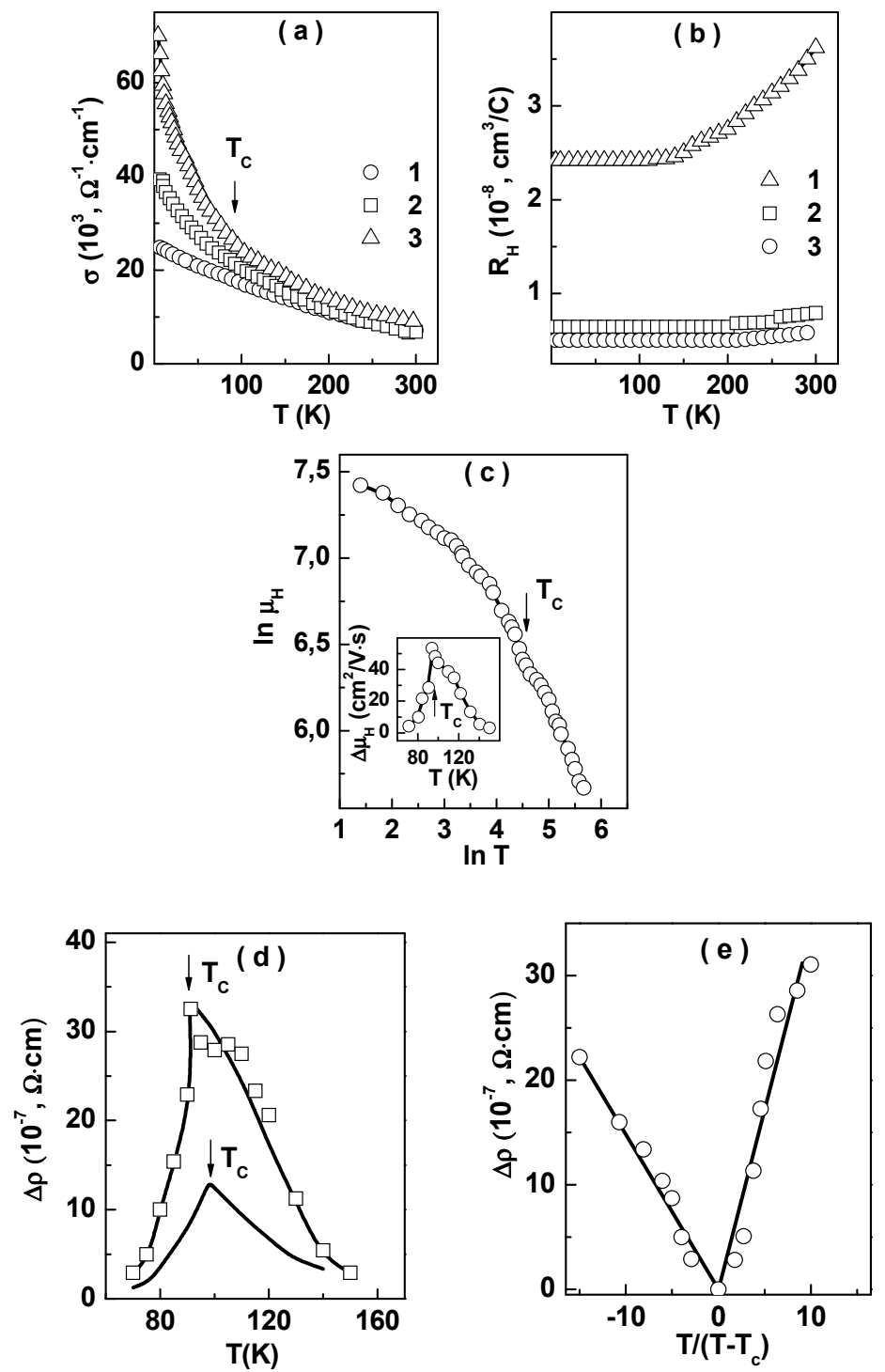

Fig. 10. Temperature dependences of the electrical conductivity $\sigma$ (a), Hall coefficient $R_{H}(\mathrm{~b})$, charge carrier mobility $\mu_{H}$ (c), additional decrease in carrier mobility $\Delta \mu_{H}$ (c) and electrical resistance $\Delta \rho$ at FPT (d,e) for SnTe with different stoichiometry. a,b: 1 - 50 at. \% Te, $2-50.4$ at.\% Te, 3 - 50.6 at. \% Te; c,d,e: 50.0 at.\% Te (Nashchekina et al., 1999b). 
There may arise a question whether the ordering processes are possible at such low temperatures. The redistribution of defects is usually associated with migration of atoms and follows the diffusion mechanism. The rate of diffusion in normal crystals at temperatures from 80 to $300 \mathrm{~K}$ is low, and such transition must be hampered. However, if diffusion occurs through the vacancy mechanism (which is the most probable in the given case), the self-diffusion coefficient $\mathrm{D}$ and the vacancy diffusion coefficient $\mathrm{D}_{\mathrm{i}}$ are connected via the relation $D=f \cdot A \cdot D_{i}$, where $f$ is the correlation factor and $A$ is the relative fraction of vacancies (Bokshtein et al., 1974; Show, 1975). Hence, an increase in the vacancy concentration leads to an increase in the self-diffusion rate. Such a sharp increase in the diffusion coefficient upon the introduction of structural vacancies caused by deviation from stoichiometry is confirmed experimentally (Show, 1975). Since the concentration of structural vacancies in SnTe at low temperatures $(\sim 1-2 \%)$ is much higher than the concentration of thermal vacancies responsible for the vacancy mechanism of diffusion in most crystals $\left(10^{-14}-10-18 \%\right.$ at $300 \mathrm{~K}$ if we assume that the energy of the vacancy formation is $E_{\mathrm{v}}=1-1.5 \mathrm{eV}$ ), we can expect that the diffusion rate in SnTe is much higher than in stoichiometric crystals. The presence of NSD of various types formed as a result of rapid cooling also stimulates the increase in the rate of diffusion processes (Bokshtein et al., 1974; Show, 1975). It should also be kept in mind that the samples were subjected to the long (200 h) homogenizing annealing at $820 \mathrm{~K}$, resulting in a high degree of crystal homogeneity and a quite uniform distribution of vacancies over the volume. Under such conditions, small displacements of atoms are sufficient for the formation of a long-range order at low temperatures. On the other hand, the possibility of defect ordering at temperatures above $300 \mathrm{~K}$ should not be ruled out either.

The observed anomalies are characterized by a considerable variation of $a$ for the same symmetry and by the negative sign of $\alpha$. This suggests that such a strong manifestation of PT is determined not only by the thermodynamic but also kinetic factors. The $a(T)$ curves were recorded during heating of the samples cooled preliminary to $80 \mathrm{~K}$ at a rate of $\sim 20 \mathrm{~K} / \mathrm{min}$. It can be assumed in this connection that a certain nonequilibrium state with $a$ higher than in the equilibrium state is established in the sample with 50.4 at.\% Te as a result of a rapid cooling and incompleteness of the diffusion processes whose rate is small at such low temperature. In other words, the structural instability of the samples with 50.4 and 50.8 at.\% Te in certain temperature intervals is caused by the presence of an equilibrium PT associated with a redistribution of NSD, but the sharp change in $a$ accompanying these PT in the process of sample heating is apparently because of the nonequilibrium nature of the initial structure formed as a result of rapid cooling from room temperature. The diffusion rate increases due to heating, and the system abruptly reaches the equilibrium state corresponding to a given temperature at the point where an equilibrium PT must take place. It should be noted that the authors of (Fukui et al., 1984) obtained amorphous SnTe films by condensation on substrates at $\mathrm{T}=4.2$ and $77 \mathrm{~K}$, and the resistivity of such films decreased in a jump-like manner upon heating at $180 \mathrm{~K}$, i.e., at the temperature close to the jump in $a$ in the $a(T)$ curve (Fig. 9,c). One should also note that the positions of two steps in the temperature dependence of the resonant field of 119Sn nucleus (Khandozhko et al., 1978) coincide with the positions of the steps in the $a(\mathrm{~T})$ curve obtained in our studies. Thus, the existence of the PT was confirmed by two different methods. 


\subsubsection{Thermal expansion coefficient}

In connection with the results of the XRD studies, it seems important to clarify how kinetic factors can affect the manifestation of the anomalies in the T-dependences of other properties. Besides, the high concentration of cation vacancies must lead to the specificity of relaxation processes, which occur in the system deviated from the equilibrium state. To additionally confirm the existence of new PT and to establish the influence of the NSD concentration on the character of the relaxation processes, we measured the $\alpha(T)$ dependences using two regimes described in 2.3.4. (Nashchekina et al, 2008). For the samples measured under the stationary regime, an increase in $\alpha$ with increasing temperature continued up to $\sim 130-140 \mathrm{~K}$ (the Debye temperature of SnTe), and the $\alpha(\mathrm{T})$ curves exhibited anomalies. In the $\alpha(T)$ curve of the sample with 50.4 at.\% Te (Fig. 11,a), an anomalous behavior is observed in the temperature ranges $140-150 \mathrm{~K}$ and $190-200 \mathrm{~K}$. The $\alpha(T)$ curves for the samples with 50.7 and 51.1 at.\% Te have plateaus at $180-190 \mathrm{~K}$ (Fig. 11,b). When measurements were carried out under the dynamic regime, the behavior of the $\alpha(T)$ curves changed significantly for all studied compositions (Fig. 11). In contrast with the stationary regime, a sharp initial increase in $\alpha$ was observed, and under the subsequent heating, the $\alpha(T)$ dependences exhibited either an oscillatory behavior (50.4 50.7 at. $\% \mathrm{Te}$ ) or a monotonic decrease in $\alpha$ with increasing temperature (51.1 at. $\% \mathrm{Te}$ ). The oscillatory behavior was most pronounced in the sample with 50.4 at.\% Te (Fig. 11,a). In the sample with the composition 51.1 at.\% Te, which is outside the HR, approaching the equilibrium state occurred in a monotonic way. For all studied compositions, starting from $220-240 \mathrm{~K}$, the curves obtained in the stationary and dynamic regimes virtually coincided (Fig. 11).

Analyzing the obtained data, one can suggest that due to quick cooling of the samples in liquid nitrogen, they get oversaturated with defects and the processes of defect redistribution in the samples are not finished. A significant deviation from the equilibrium is confirmed by the sharp increase in $\alpha$ measured in the dynamic regime compare to the values of $\alpha$ obtained in the stationary regime, which was clearly observed in all samples (Fig. 11). With increasing Te concentration, a growth in $\alpha$ caused by immersing the sample in liquid nitrogen becomes more significant, which indicates that the degree of disordering in the system increases. Under heating of such nonequilibrium system, due to an increase in the diffusion rate, which leads to the defect movement and partial annihilation of defects, complex processes must take place. These processes are caused, on the one hand, by the relaxation of the system towards the equilibrium state and decrease in $\alpha$, and on the other hand, by phase transformations occurring in a system whose state is far from equilibrium. Such processes can lead to a gigantic lattice instability in the vicinity of the temperature of a PT (equilibrium or nonequilibrium). When returning to the equilibrium state, the system can pass through a number of intermediate states corresponding to metastable equilibria at given temperatures. As a result, the character of the $\alpha(T)$ dependences becomes more sophisticated. The fact that values of $\alpha$ measured under the two above described regimes coincide at temperatures above $\sim 240 \mathrm{~K}$ shows that in the vicinity of $240 \mathrm{~K}$ the system returns to the equilibrium and that the state of the defect subsystem is identical for both regimes. As is seen from Fig. 11, the character of the relaxation processes and kinetics of the process of reaching equilibrium in the crystal defect 
subsystem depend on the cation vacancy concentration. The $\alpha(T)$ dependences for most of the studied samples exhibit an oscillatory behavior, although locations, amplitudes and nature of oscillations vary. The oscillations are most pronounced for the sample with 50.4 at.\% Te, and with increasing Te concentration these oscillations become less distinct (Fig. 11). One can suggest that these oscillations are associated mainly with the defect redistribution processes (PT) taking place under nonequilibrium conditions. That is why the oscillations observed in the $\alpha(\mathrm{T})$ curve of the sample with 50.4 at.\% Te measured under the dynamic regime (Fig. 11) can be connected with a high probability of the redistribution of NSD which takes place in a system whose state is far from the thermodynamic equilibrium. A larger magnitude of the anomaly at 190-200 K observed in the sample with 50.4 at. \% Te under the dynamic regime compare to the stationary regime is apparently caused by such redistribution. For the samples with 50.5-50.8 at.\% Te, the $\alpha(T)$ dependences are similar but the manifestation of the anomalies is less pronounced. The most probable reason for that is deviation from the optimum ordering composition and, as a result, diminishing stimuli for ordering. However, the increase in $\alpha$ in comparison with its equilibrium value becomes more noticeable, and an oscillatory character of the relaxation processes manifests itself more distinctly. To all appearances, the oscillatory character of the relaxation processes is connected to a great extent with a high concentration of NSD, whose presence leads to loosening of the crystal lattice, slackening of bonds and decrease in the period of oscillations, thus making them observable.

\subsubsection{Electronic properties}

The temperature dependences of $R_{H}, \mu_{H}, \sigma$ in the range 77 - $300 \mathrm{~K}$ were obtained for SnTe epitaxial and polycrystalline thin films with different thicknesses $d(0.2-2.0 \mu \mathrm{m})$ and different carrier concentrations: $p_{\mathrm{H} 77}=3.5 .10^{26}, 4.5 \times 10^{26} \mathrm{~m}^{-3}$, and $p_{\mathrm{H} 77}=1 \times 10^{27} \mathrm{~m}^{-3}$ (Nashchekina \& Rogacheva, 1998). Techniques were developed for the preparation of monoand polycrystalline SnTe films with controlled concentration of NSD and high $\mu_{\mathrm{H}}$ (Nashchekina et al, 1999a; Nashchekina et al, 1995). The best results were attained using the hot wall method, which permits to vary the cation vacancy concentration in films, and thus their properties, in a wide range. Analysis of the temperature dependences of kinetic characteristics showed that the values of mobility in mono- and polycrystalline films are rather close and comparable to those in bulk crystals. It was established that the exponent in the $\mu_{H}(T)$ relationship is determined by the hole concentration, temperature, as well as by the degree of the film structure perfection, and its values lie in the range from 0 to 0.7 . The highest values of the exponent are found in monocrystalline films with low $p$ at low temperatures.

For the epitaxial SnTe films with $p_{\mathrm{H} 77}=3.5 \cdot 10^{26} \mathrm{~m}^{-3}$ and thicknesses $d=0.2$ and $0.4 \mu \mathrm{m}$ in the temperature range $180-200 \mathrm{~K}$, an increase in $R_{H}$ and a pronounced slowing down of the drop in $\mu_{H}$ are observed (Fig 12). For the sample with $d=0.6 \mu \mathrm{m}$, in the vicinity of 200 $\mathrm{K}$, pronounced anomalies of $R_{H}$ and $\mu_{H}$ are registered representing evidence of the qualitative changes in the electron and phonon spectra. The values of $\mu_{H}$ for this sample are higher than those for the other two. One can suggest that the anomalies in the $R_{H}(\mathrm{~T})$ and $\mu_{H}(\mathrm{~T})$ dependences in the vicinity of $200 \mathrm{~K}$ are caused by the PT connected with a redistribution of NSD. The high mobility and the high, for such films, value of $b$ below 200 
$\mathrm{K}$ indicate the possibility of the formation of an ordered structure in which electron scattering by defects becomes insignificant due to their regular distribution over the crystal lattice. The sharp character of transition in the sample with $d=0.6 \mu \mathrm{m}$ and the gradual one in the samples with $d=0.2$ and $0.4 \mu \mathrm{m}$ can be associated with kinetic factors determining scale and degree of ordering. At small $d$, the ordering processes can be hampered due to a considerable contribution of the surface energy. For the investigated films, the thickness of $0.6 \mu \mathrm{m}$ seems to be optimal for ordering. One can assume that the increase in $R_{H}$ above $200 \mathrm{~K}$, interpreted usually in the framework of the two-band model, is connected with a disordered distribution of NSD and/or the appearance of defects of a new type. In Fig. 12,a, the $R_{H}(T)$ and $\mu_{H}(T)$ dependences for the sample with $p=4.5 \times 10^{26}$ $\mathrm{m}^{-3}$ are shown. As is seen, there are two distinct anomalies in the temperature ranges 135150 and 200-215 K. In Fig. 12, the $\rho(T)$ dependences for polycrystalline SnTe films $(d=0.27$ and $2.0 \mu \mathrm{m}$ ) with $p=(3-4) \cdot 10^{27} \mathrm{~m}^{-3}$ deposited on glass substrates at room temperature are presented. In the film with $d=0.27 \mu \mathrm{m}, \rho$ increases practically linearly with increasing temperature, and in the region 135-145 $\mathrm{K}$ a horizontal section is observed, whereas in the $2 \mu \mathrm{m}$ thick film there are two anomalous sections in the temperature ranges $145-155 \mathrm{~K}$ and 185-200 K. It follows from the obtained data that the temperature intervals in which the anomalies of $\rho, R_{H}$ and $\mu_{H}$ are observed are practically the same $(135-150 \mathrm{~K}, 185-215 \mathrm{~K})$ for both mono- and polycrystalline films. It is seen that the reconstruction of the defect subsystem is accompanied not only by a sharp ( 30\%) drop in mobility but also by a noticeable change in $R_{H}(15-20 \%)$. Let us note that under the FPT no change in $R_{H}$ was observed, i.e. insignificant changes in the crystal lattice symmetry do not practically affect the electronic subsystem. On the other hand, the processes of the defect redistribution accompanied by a change in the electronic structure of defects, in the character of chemical bonds in the vicinity of a defect, which determine the number of charge carriers and their mobility, cause changes not only in $\mu_{H}$ but also in $R_{H}$. The fact that under certain conditions the ordering processes are more easily realized in thin films is by no means exceptional. In a large number of III-V alloys with isovalent substitution, the phenomenon of atomic scale ordering during film growth, which is not thermodynamically stable in the bulk alloys, has been revealed. The probability of cation vacancy ordering in SnTe films increases due to the effect of such factors as the Coulomb repulsion between charged defects and a large deformation energy connected with their formation. As is seen from the results obtained, the realization of these processes is controlled by kinetic factors. In bulk crystals the diffusion processes are inhibited, which prevents the defect redistribution and formation of a configuration corresponding to the minimum of free energy. A detailed study of the T-dependences of $\rho$ and $S$ for the SnTe polycrystalline films with $p=(3-5) \cdot 10^{27} \mathrm{~m}^{-3}$ was conducted in the range of $80-300 \mathrm{~K}$ (Rogacheva et al, 2003c). In the $\rho(T)$ and $S(T)$ curves within the range 80-150 K a number of peculiarities in the form of steps and plateaus were exhibited most distinctly. The possible reasons for the appearance of the temperature peculiarities are the system's passing through different quantum states; the processes of self-organization taking place in an open system; microdomain structure of thin films; relaxation processes. The pronounced anomalies observed in the ranges of $135-150$ and $190-200 \mathrm{~K}$ are attributed to PT caused by the redistribution of NSD. 

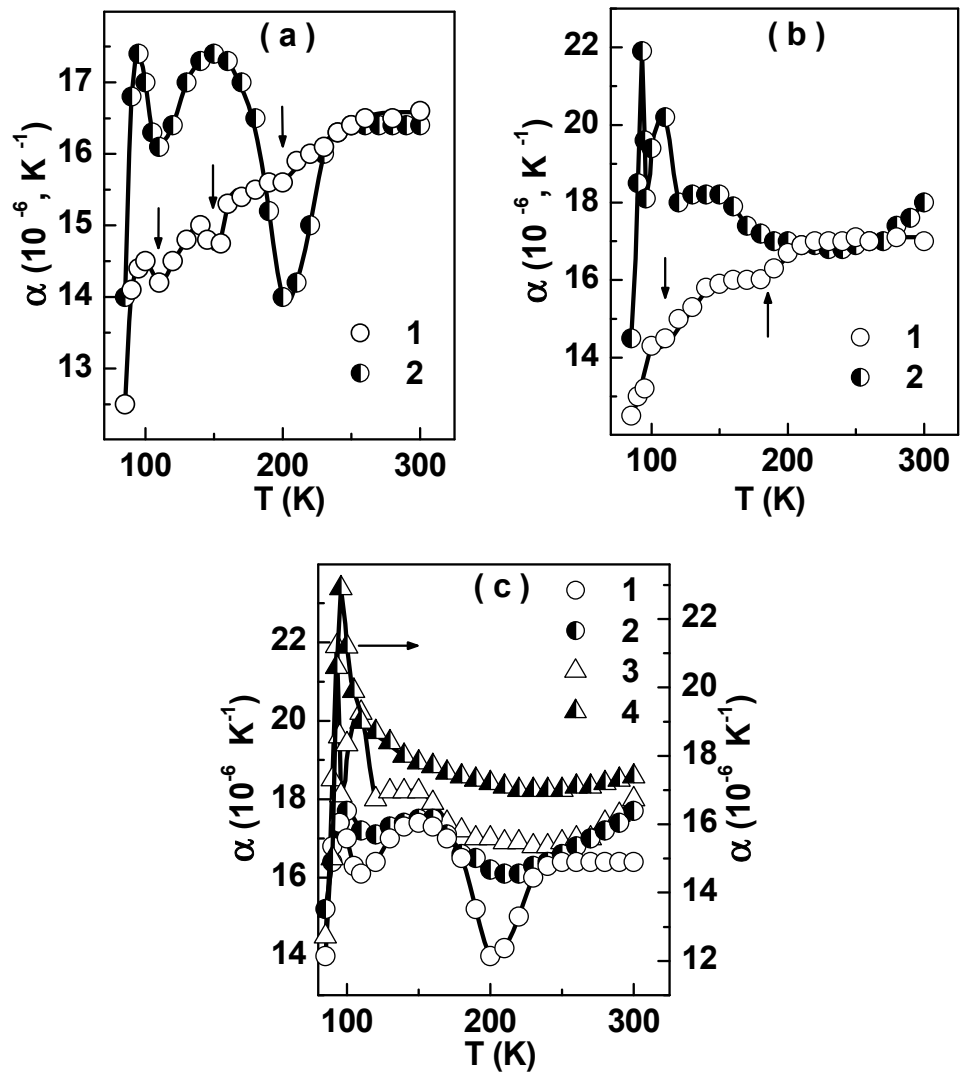

Fig. 11. Temperature dependences of the thermal expansion coefficient $\alpha$ for SnTe with 50.4 (a) and 50.7 (b) at.\% Te (1 - stationary regime; 2 - dynamic regime) and $\alpha(T)$ dependences for SnTe with different stoichiometry obtained under a dynamic regime (1 - 50.4 at.\% Te, 2 50.5 at. \% Te , 3 - 50.7 at.\% Te, 4 - 51.1 at.\% Te) (Nashchekina et al, 2008).

\section{Problems of doping of non-stoichiometric SnTe}

\subsection{Mechanism of impurity dissolution in nonstoichiometric SnTe}

One of the basic ways of controlled changing of properties of semiconductors is doping, which is accompanied by a partial disordering of the structure by IA. It is impossible to develop physical principles for controlled doping of NS phases without the knowledge of the specific physical effects connected with the simultaneous introduction of IA and NSD in a crystal. IA can be introduced into a NS compound in the elementary form or in combination with other components of the host compound, for example, by cation substitution. In the Sn-Te-X (X is the impurity component) concentration triangle, different schemes of the IA introduction correspond to changing the alloy composition along a certain section passing through SnTe compound. We will limit our consideration to two doping schemes - the introduction of elementary impurities and the introduction of IA by cation substitution. 

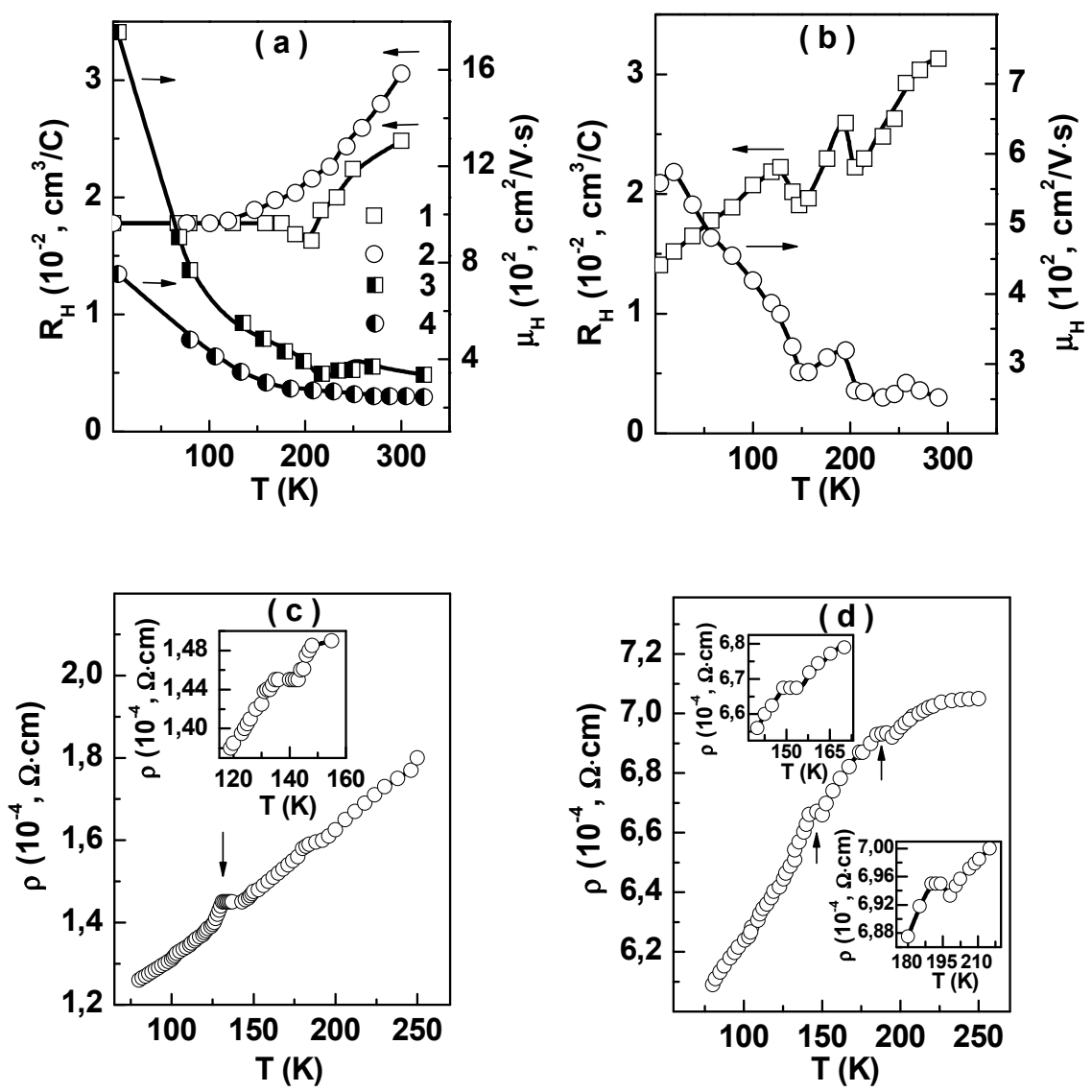

Fig. 12. Temperature dependences of Hall coefficient $R_{H}(\mathrm{a}, \mathrm{b})$, charge carrier mobility $\mu_{H}$ $(\mathrm{a}, \mathrm{b})$, and electrical resistance $\rho(\mathrm{c}, \mathrm{d})$ for SnTe thin films deposited on $\mathrm{KCl}$ at $520 \mathrm{~K}(\mathrm{a}, \mathrm{b})$ or glass at $300 \mathrm{~K}(\mathrm{c}, \mathrm{d})$ substrates with carrier concentration $p_{\mathrm{H} 77}=3.5 \cdot 10^{26} \mathrm{~m}^{-3}(\mathrm{a}), 4.5 \cdot 10^{26} \mathrm{~m}^{-3}$ (b), $4.0 \cdot 10^{27} \mathrm{~m}^{-3}$ (c), 3.0.1027 $\mathrm{m}^{-3}$ (d) and with different thicknesses d (Nashchekina et al.,1999a, 1995; Nashchekina \& Rogacheva, 1998). a: 1, 3- $d=0.6 \mu \mathrm{m}, 2,4-d=0.2 \mu \mathrm{m} ; \mathrm{b}: d=0.4 \mu \mathrm{m}$; :

$d=0.27 \mu \mathrm{m} ; \mathrm{d}: d=2 \mu \mathrm{m}$

The basic mechanisms of the impurity dissolution in a stoichiometric semiconductor compound are the following: i) IA enter interstices and ii) IA fill the basic structural sites in one sublattice at the simultaneous formation of vacancies in the other one. To establish the mechanisms of IA dissolution in a crystal with NS vacancies, we performed a complex of studies (Dzyubenko et al., 1981,1983; Rogacheva \& Dzyubenko, 1986; Rogacheva et al., 1985,1988 ) of the dependences of properties on the IA concentration at a fixed content of intrinsic defects. $\mathrm{Sn}_{0.984} \mathrm{Te}$ was chosen as the initial composition, and thus the crystal lattice contained $\sim 1.6$ at. $\%$ of vacant cation sites. The analysis of the concentration dependences of properties (Fig. 13) shows that there are two types (I and II) of impurities. For impurities I 
(In, Ga, Cd, etc.), a nonmonotonic behavior of the concentration dependences of properties is observed, which suggests that under changing IA concentration different mechanisms of IA dissolution are realized (Fig. 13, a). At $0.1-0.2$ at.\% of impurity, extreme points or bends are observed, which divide HR into two subregions with different character of the concentration dependences of properties. In the subregion of small IA concentrations, the sharp increase in $H, p, a$, and the decrease in $\mu_{H}$ and $\lambda_{p}$ occur with increasing IA concentration. In the second subregion, $H$ and $p$ decrease, $\mu_{H}$ and $\lambda_{p}$ increase as the IA concentration increases. For impurities II, the behavior characteristic only of the second subregion is observed (Fig. 13, b).

The sharp increase in $H$ and $a$ at small concentrations of impurity atoms evidences that a significant deformation of the crystal lattice takes place, whereas the decrease in $\mu_{H}$ and $\lambda_{p}$ is connected with increasing hole and phonon scattering by IA. The extreme character of the concentration dependence of $p$ can be attributed to the change in the electrical action of IA from acceptor to donor under increasing IA concentration, which indicates a reconstruction of chemical bonds in the vicinity of IA. In this connection one can suggest that the introduction of the first portions of IA into the crystal lattice takes place by the interstitial mechanism, and IA are localized in the tetrahedral interstices of the close packing of Te atoms. In semiconductor crystals, which usually have relatively small coordination numbers and rather loose structure, the enthalpy of the IA formation is comparable with the enthalpy of the vacancy formation, and that is why interstitials play more important role in semiconductors than in metals.

The behavior of the properties in the range of IA concentrations greater than $0.1-0.2$ at. \% for impurity I or within the entire HR for impurity II suggests that the main mechanism of the IA dissolution is the filling of cation vacancies. The decrease in $a$ observed in SnTe under increasing deviation from stoichiometry (Fig. 13,a) indicates the compression of the crystal lattice. A vacancy creates tensile strains around the defect, and the introduction of an IA leads to partial compensation of these strains. The decrease in $H$ shows that the overall level of strains in the crystal lattice decreases. The main parameters that determine the degree of change in $H$ under the introduction of point defects are the energies of elastic and electrical interaction between the defect and dislocation. Since the energy of elastic interaction is proportionate to the change in volume under the substitution of the host atom by the defect, the rate of change in $H$, occurring when the IA fills the vacancy, must depend somehow on the difference between the cation and vacancy radii. The comparison of the rate of the decrease in $H$ when IA fill vacancies for impurities with the same valence showed that with decreasing difference in the radii of the impurity cation and cation vacancy this rate decreases (Dzubenko et al, 1983). IA localized in vacancies give their valence electrons to Te thus reducing the overall concentration of holes. The reduction in the crystal lattice distortions under the filling of vacancies leads to a reduction in phonon and electron scattering and consequently to an increase in $\mu_{H}$ and $\lambda_{p}$. It is established that all elementary impurities introduced into $\mathrm{Sn}_{0.984}$ Te reduce $p$, simultaneously reducing $S$. The exception is In, which exhibits variable valence. In case of In impurity, $S$ increases with decreasing $p$.

A question arises whether the observed effect related to a change in the localization of IA (from interstitials to cation vacancies) is an equilibrium one or corresponds to a metastable state produced by incomplete diffusion. In this connection we investigated the effect of thermal treatment on the concentration dependence of $H$ and $a$ in SnTe doped with Cd, In, 
and $\mathrm{Sb}$ (Rogacheva et al., 1985). In particular we applied annealing at 820K for $120 \mathrm{~h}$ (HT1) and for $420 \mathrm{~h}$ (HT2) and aging at room temperature for 2 years (HT3). In all samples subjected to HT1, a sharp increase in $H$ and $a$ was observed under the introduction of impurity up to 0.1-0.2 at. \%, and then for $a$ the rate of the increase decreased and for $H$ it sharply dropped (Fig. 14). After HT2 in the systems doped with Sb, $H$ decreased monotonically in the entire solubility region, and $a$ increased at a lower rate than after HT1. In the systems doped with $\mathrm{Cd}$ and In, the general behavior of the dependences of $\mathrm{H}$ on composition did not change, although the relative increase in $H$ and $a$ at small concentrations decreased. HT3 caused practically no qualitative changes in the concentration dependences of the properties. Thus, at small IA content, the character of the property-composition dependences is controlled to a great extent by diffusion processes, and in a number of cases, the fixation of nonequilibrium states is possible. The stability of these states depends upon the IA diffusion coefficients. Thus, the presence of NSD in SnTe leads to the appearance of a new mechanism of IA dissolution consisting in the occupation of vacancies by IA. In case of some impurities (In, Ga, etc.), at small concentrations (< 0.2 at. $\%)$, IA enter tetrahedral interstices, and the mechanism is controlled by diffusion processes.

\subsection{Solubility limits of impurities in SnTe}

The limits of solubility for different elements in SnTe at fixed concentrations of intrinsic defects were determined on the basis of the results of the microstructure studies and measurements of the concentration dependences of properties after homogenizing annealing of samples at $820 \mathrm{~K}$ for 300 hours. $\mathrm{Sn}_{0.984} \mathrm{Te}$ was chosen as the initial composition. The analysis of the obtained results shows that the solubility limits $\left(C_{\text {lim }}\right)$ for different elements differ substantially. When vacancies are occupied by IA, defects of a new type impurity substitution defects - are created in the crystal lattice in addition to vacancies, which causes the appearance of elastic stress fields because of the difference in the cation sizes and violates the periodicity in the distribution of the crystal lattice potential because of the difference in the electronic configurations of $\mathrm{Sn}$ and impurity atoms. It is therefore natural to expect that $C_{\text {lim }}$ depends both on the crystallochemical radii of $S n$ and IA and on their valence states. Fig. 15, a shows the dependences of $C_{\lim }$ on the relative difference $\left(\Delta r / r_{S n}\right.$ $\left.=\left(r_{i m p}-r_{S n}\right) / r_{S n}\right)$ of the ionic radii of the impurity $\left(r_{i m p}\right)$ and $S n\left(r_{S n}\right)$ atoms (Rogacheva \& Dzubenko, 1986). It is evident from Fig. 15 that there is a correlation between $C_{\text {lim }}$ and $\Delta r / r_{S n}$ both for positive and for negative values of the size factor: when $\Delta r / r_{S n}$ increases, the solubility of the impurity decreases. The different slopes of the straight lines for positive and negative values of $\Delta r / r_{S n}$ for SnTe show that the compression and tensile deformations, appearing when the vacancies are filled by cations larger or smaller than Sn, affect the stability of the crystal lattice in different ways. For the same relative difference in the ionic radius, when $\mathrm{Sn}$ ions are replaced by smaller cations, the value of $\mathrm{Clim}_{\mathrm{lim}}$ is higher than in case of substitution by larger cations. The influence of the vacancy concentration in SnTe on the solubility of IA was investigated in (Rogacheva et al, 1988; Vodoriz, 2009). It was established that the solubility of $\mathrm{Sb}$ in SnTe increases linearly with an increase in the concentration of cation vacancies up to $\sim 1$ at. $\%$ (Rogacheva et al, 1988) (Fig. 15,b). When IA are introduced in a NS phase with a vacancy concentration exceeding $\sim 1$ at. $\%$, chemical interaction between impurity and host atoms in the solid solution becomes an additional factor affecting the solubility limit and character of the change in properties (Rogacheva et al., 1988). Similar results were obtained for SnTe doped with Cu (Vodoriz et al., 2009). 

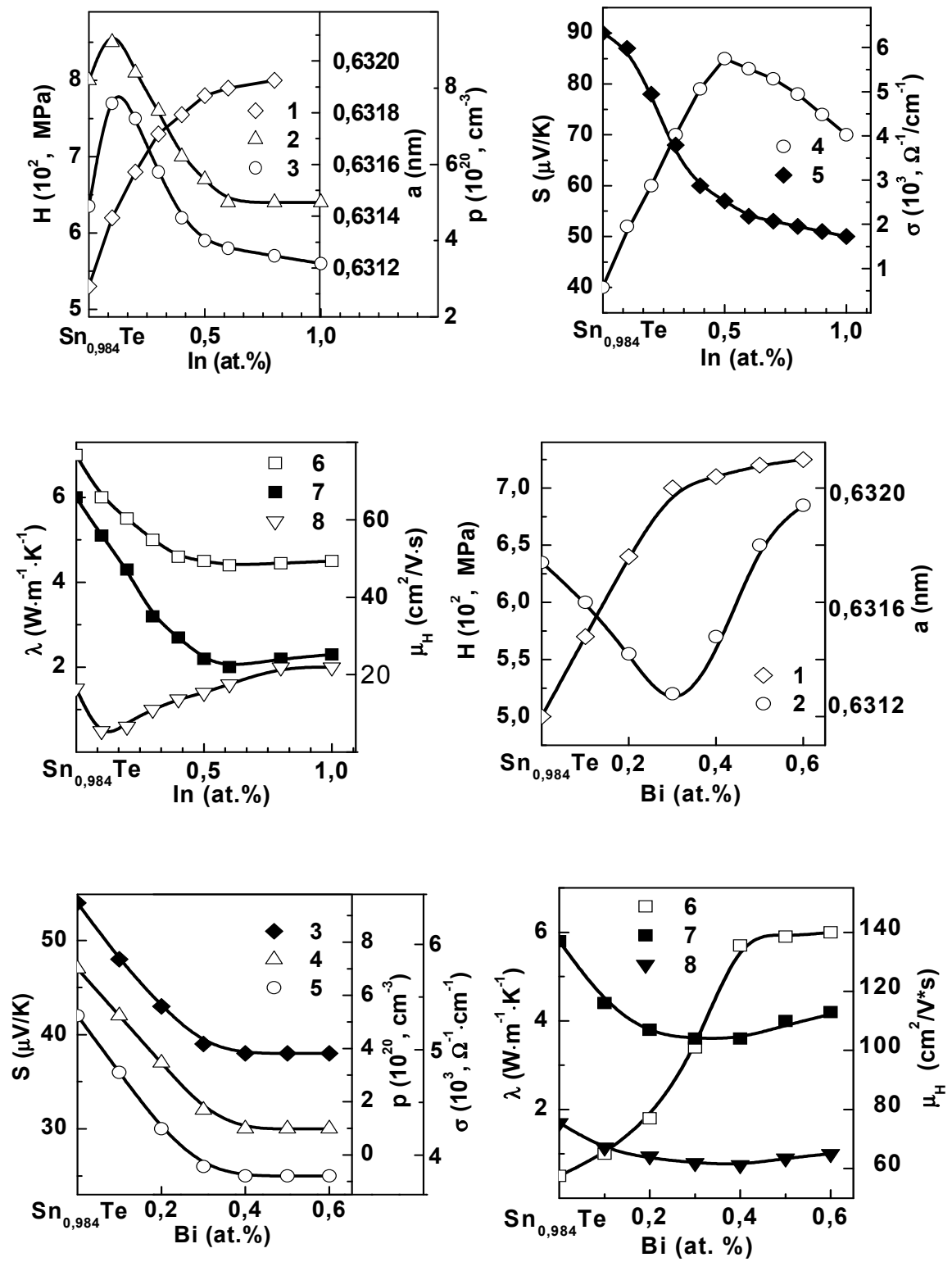

Fig. 13. Unit-cell parameter $a$, microhardness $H$, carrier concentration $p$, Seebeck coefficient $S$, electrical conductivity $\sigma$, total thermal conductivity $\lambda$, lattice thermal conductivity $\lambda_{p}$, and Hall carrier mobility $\mu_{H}$ of SnTe as a function of In and Bi content. In: $1-\mathrm{a} ; 2-\mathrm{p} ; 3-\mathrm{H} ; 4-\mathrm{S}$; $5-\sigma ; 6-\mu_{H} ; 7-\lambda ; 8-\lambda_{p} ;$ Bi: $1-\mathrm{a} ; 2-\mathrm{H} ; 3-\sigma ; 4-\mathrm{p} ; 5-\mathrm{S} ; 6-\mu_{\mathrm{H}} ; 7-\lambda ; 8-\lambda_{\mathrm{p}}$. (Dzubenko et al., $1981,1983)$ 

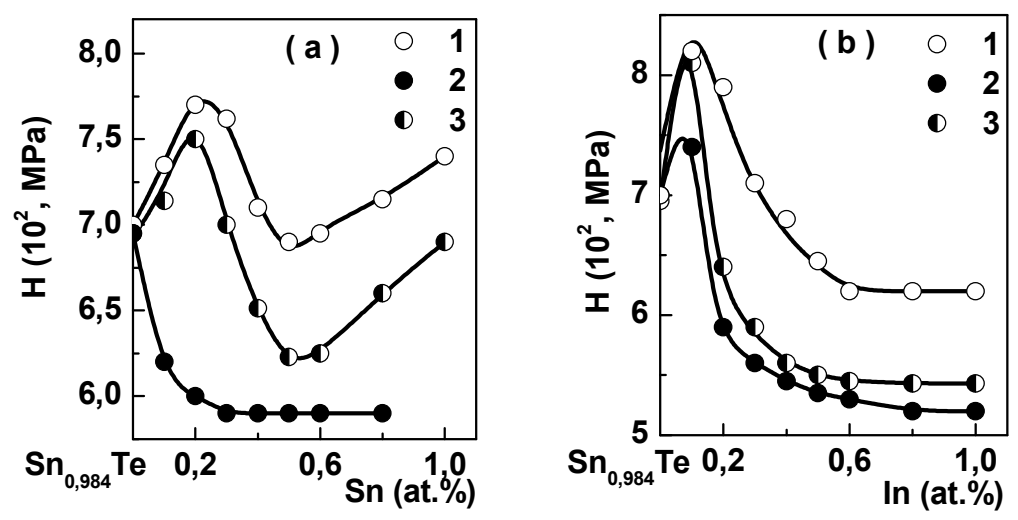

Fig. 14. The dependences of microhardness $H$ on $\mathrm{Sb}(\mathrm{a})$ and In (b) concentration in $\mathrm{Sn}_{0.984} \mathrm{Te}$

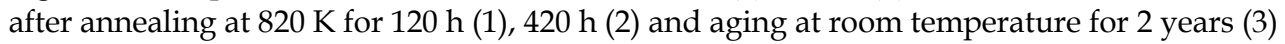
(Rogacheva et al., 1985).

\subsection{The influence of a third component on the equilibrium of intrinsic defects in SnTe}

The introduction of a third component $X$ in a binary compound must lead to a change in the thermodynamic equilibrium conditions and to a change in the intrinsic defect concentration (Kröger, 1973). One can judge about the character of this change by the position of the boundaries of HR of a SnTe-based phase in a ternary Sn-X-Te system. At a fixed $\mathrm{T}$, such HR represents a two-dimensional region within the solubility isotherm. We plotted the solubility isotherms using a wide array of experimental methods (Rogacheva, 2005). It was established that the solubility isotherms have a pronounced singular character and the direction of maximum solubility corresponds to the cross-section passing through the stable compound formed in an X-Te system. In the systems with $\mathrm{Cu}$ and $\mathrm{Ag}$, the isotherms are oriented in the direction of $\mathrm{Cu}_{2} \mathrm{Te}$ and $\mathrm{Ag}_{2} \mathrm{Te}$ compounds, respectively, while in the systems with $\mathrm{Sb}$ and $\mathrm{Bi}-$ in the direction of $\mathrm{Sb}_{2} \mathrm{Te}_{3}$ and $\mathrm{Bi}_{2} \mathrm{Te}_{3}$ (Fig. 16). The observed regularities can be qualitatively predicted on the basis of the active mass law (Kröger, 1973; Glazov \& Pavlova, 1988): there is a dynamic equilibrium between the concentrations of ionized intrinsic and impurity defects, the presence of charged impurity centers leads to an increase in the concentration of intrinsic defects of the opposite sign. The analysis of the solubility isotherms allowed us to reveal another interesting effect: in all isotherms in the range of small IA concentrations (up to $\sim 0.5-1.0$ at.\%), a shift of the SnTe HR boundary in the direction of increasing degree of deviation from stoichiometry is observed. The formation of additional vacancies in the diluted solid solutions can be stimulated by such factors as a decrease in the energy of vacancy formation in a strongly distorted crystal, a larger increase in the configuration entropy under introduction of IA into a crystal with vacancies, and other factors. We attribute the bents in the solubility isotherms in the range of small IA concentrations to a transition from a weak doping when the interaction between IA can be neglected to a heavy doping when this interaction becomes cooperative. 

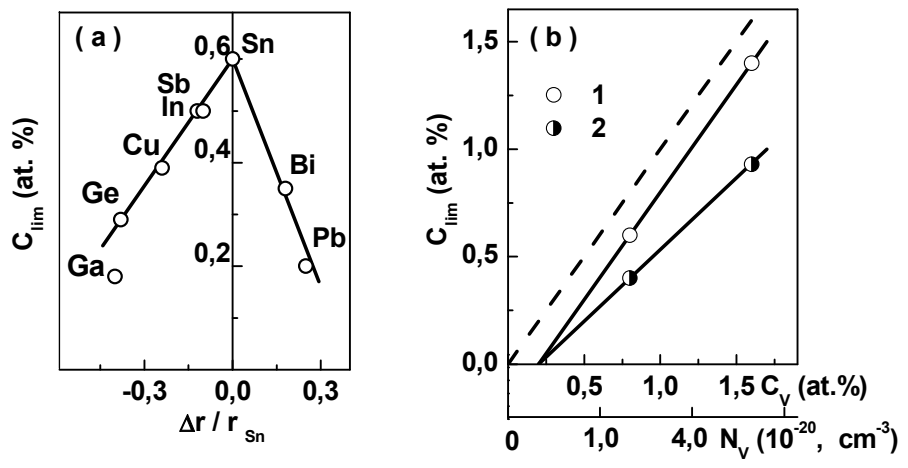

Fig. 15. The dependences of the solubility limit $\left(C_{\text {lim }}\right)$ of impurities in SnTe on the relative difference in the ionic radii of impurity and Sn atoms $\left(\Delta r / r_{S_{n}}=\left(r_{i m p}-r_{S_{n}}\right) / r_{S_{n}}\right)$ (a) (Rogacheva\&Dzubenko, 1986) and the dependence of the solubility limit of Sn (1) and Sb (2) on the concentration of cation vacancies in SnTe (b) (Rogacheva et al., 1988).
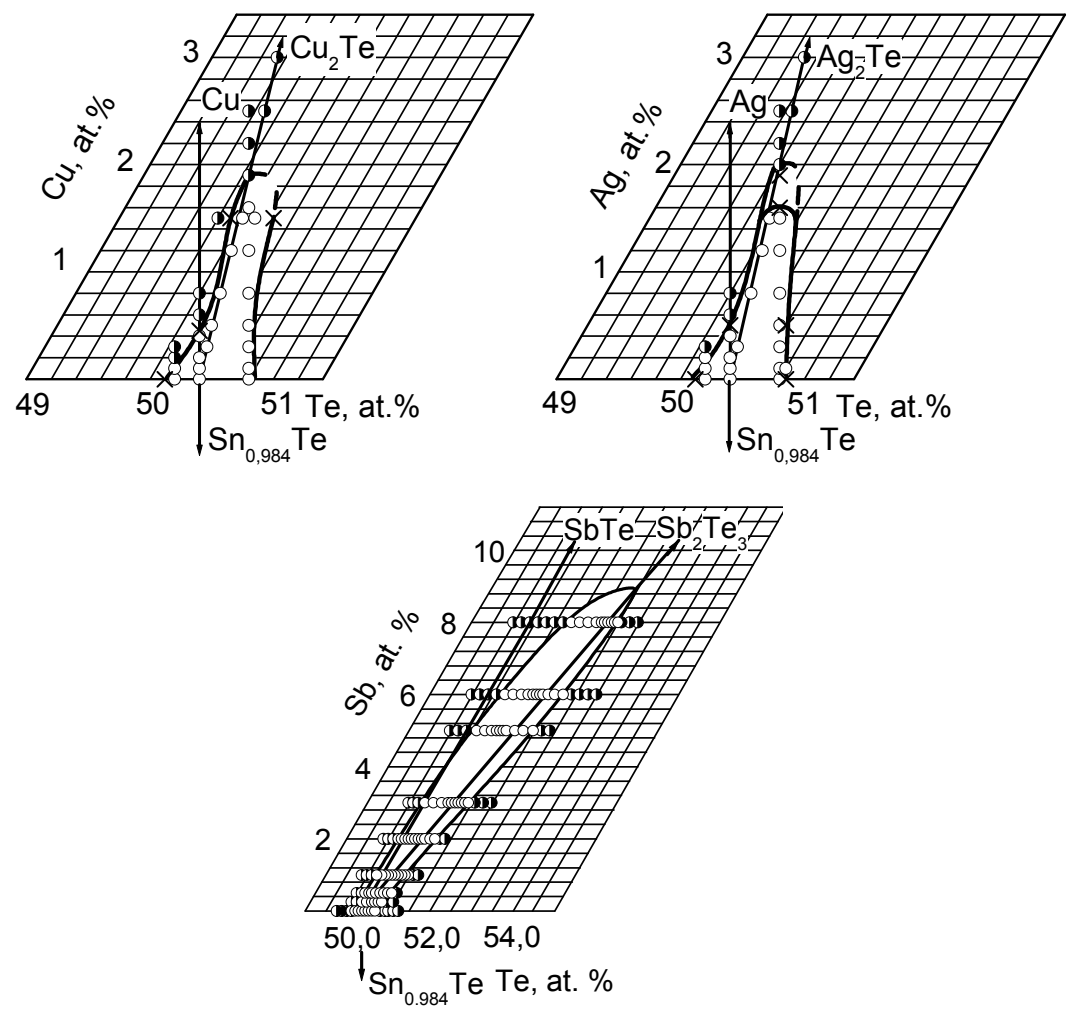

Fig. 16. Isotherms of solubility at $520 \mathrm{~K}$ in the $\mathrm{Sn}-\mathrm{Cu}-\mathrm{Te}, \mathrm{Sn}-\mathrm{Ag}-\mathrm{Te}$, and $\mathrm{Sn}-\mathrm{Sb}-\mathrm{Te}$ ternary systems (Rogacheva, 2005). 


\subsection{Impurities with variable valence and nonstoichiometric vacancies in SnTe}

Under the introduction of cations with variable valence into SnTe, an unusual effect connected with the possibility of the formation of chemical bonds of different types in SnTebased solid solutions was detected (Rogacheva et al., 1983,1991,2003d; Baltrunas et al., 1988; Gorne et al., 1987). The effect consists in an anomalous influence of deviation from stoichiometry on properties of SnTe doped with In. The movement along In isoconcentrates in the Sn-Te- In ternary system in the direction of increasing Te concentration leads to the recharging of In cations $\left(\mathrm{In}^{1+} \rightarrow \mathrm{In}^{3+}\right)$, compensation of the acceptor action of vacancies by the donor action of $\mathrm{In}^{3+}$ and a decrease in the hole concentration, which continues up to the intersection with the $\mathrm{SnTe}-\mathrm{In}_{2} \mathrm{Te}_{3}$ section. It was shown that the change of the charge state of In atoms is associated with a change in their localization in the crystal lattice. The model of energy spectrum of SnTe doped with In was proposed (Rogacheva \& Laptev, 1999). According to the model, the valence band contains two resonance bands corresponding to $\mathrm{In}^{1+}$ and $\mathrm{In}^{3+}$. The vacancy band is the lowest at the energy scale of the valence band, followed by $\mathrm{In}^{1+}$ and $\mathrm{In}^{3+}$ bands (Fig. 8,b). The width of the $\mathrm{V}_{0}$ or $\mathrm{In}^{1+}$ subbands and effective mass of holes are determined by the distance between defects. In (Rogacheva et al, 1997), on the basis of the Mössbauer measurements it was shown that introducing $\mathrm{Ag}_{2} \mathrm{Te}$ into $\mathrm{Sn}_{0.984} \mathrm{Te}$ results in partial change of $\mathrm{Sn}$ valence from $\mathrm{Sn}^{2+}$ to $\mathrm{Sn}^{4+}$.

\section{Conclusions}

The specificity of chemical bonds and defect structure of semiconductors in comparison with metals determines the necessity of further development of the concept of a nonstoichiometric phase. Semiconductor compounds with one-sided wide homogeneity region represent a special group of nonstoichiometric phases. We discussed the specificity of properties of such phases using SnTe as an example. The conducted studies yielded the following results.

- It was established that as temperature decreases, the SnTe HR boundaries shift towards the composition 50.4 at.\% Te, corresponding to the maximum in melting curves. This composition is marked with special points in the concentration dependences of different properties. The possibility of the formation of vacancies superstructure at 50.4 at.\% Te is suggested.

- It was shown that it is fruitful to use the percolation theory approach for studying properties of phases with wide HR.

- A new approach to studying the genesis of energy band spectrum of semiconductor phases with wide HR, taking into account a high concentration of NS defects, was proposed, and a principally new model of energy band spectrum was developed for SnTe and for SnTe doped with indium.

- It was shown that the presence of NS vacancies in the host compound leads to the appearance of a new mechanism of impurity dissolution consisting in the occupation of vacancies by impurity atoms. The solubility limits for different elements in SnTe at fixed concentrations of NS cation vacancies were determined. At vacancy concentrations less than $\sim 1$ at.\%, there is correlation between solubility limit and relative difference in ionic radii of $\mathrm{Sn}$ atoms and those of the impurity. The solubility limit increases with increasing concentration of NS vacancies in the host material. 
- It was revealed that the introduction of cations with variable valence ( $\mathrm{In}, \mathrm{Cu}$ ) leads to a specific effect caused by the presence of NS vacancies and consisting in the recharging of cations under changing concentration of impurity atoms or deviation from stoichiometry.

- For SnTe, new temperature phase transitions associated with redistribution of NS defects were revealed.

- There was proposed a classification of one-sided phases, the composition of a phase at $\mathrm{T}=0$ being chosen as a main criterion. "Genuine NS phases" remain one-sided in the entire temperature range, even at $\mathrm{T} \rightarrow 0$. The formation of such phases can be connected with a decrease in the crystal free energy under deviation from stoichiometry due to an increase in the contribution of the electron subsystem to the crystal total energy and defect ordering.

\section{Acknowledgments}

The author thanks Dr. Olga Nashchekina for fruitful and stimulating discussions.

\section{References}

Abrikosov, N.Kh., \& Shelimova, L.E. (1975). Semiconducting materials based on $A^{4} B^{6}$ compounds, Nauka, Moscow.

Allgaier, R.S. \& Sheie, P.O. (1961). Electrical properties of p-type SnTe. Bull. Am. Phys. Soc., vol. 6, No. 2, (February, 1961), pp. 436-437, ISSN 0003-0503.

Akchurin, R.Kh., \& Ufimtsev, V.B. (1979) Calculation of lead and tin tellurides homogeneity regions boundaries. Zhurnal fizich. khimii, Vol. 53, No. 6, (June, 1979), pp. 1441-1445, ISSN 0044-4537.

Andreev, A.A. (1967). Parameters of the two valence bands model for SnTe from Hall effect measuring at high temperature. Fizika tverdogo tela, Vol. 9, No. 5, (May, 1967), pp. 1560-1561, ISSN 0367-3294.

Baltrunas, D., Motiejunas, S., \& Rogacheva, E.I. (1986). Effect of the Deviation from Stoichiometry on the Mossbauer Parameters of SnTe. Phys. Stat. Solidi. A, Vol. 97, No. 2, (February, 1986), pp. K131-K133, ISSN 1862-6319.

Baltrunas, D.I., Gorne, G.V., Laptev, S.A., Moteyunas, S.V., \& Rogacheva, E.I. (1988). Nature of SnTe-based solid solutions in the system SnTe-InTe. Izv. AN SSSR. Neorgan. Material, Vol. 24, No 10, (1988), pp. 1398-1401.

Bashkirov, Sh.Sh., Dobrjakov, I.A., Liberman, A.B., \& Tsarevskiy, S.S. (1985). Mossbauer and $X$-ray investigations of the high-temperature phase transition in SnTe. Kristalographiya, Vol. 30, No. 6, , pp. 1016-1017, ISSN 0023-4761.

Bekker, A.A., Zhukov, Yu.M., Kaukis, A.A., \& Nesmeyanov, A.N. (1973). Tin telluride study using gamma-resonance spectroscopy method. Vestnik Moskovskogo Univ., Ser. Khimiya, No. 4, (1973), pp. 434-436, ISSN 0579-9384.

Belson, H.S., \& Houston, B. (1970). Thermal expansion of tin telluride. J. Appl. Phys., Vol. 41, No. 1, (January- February, 1970), pp. 422-424, ISSN 0021-8979.

Berman, R. (1979). Thermal Conduction in Solids, Mir, ISBN 1704060000, Moscow, (in Russian).

Bokii, G.B., (1971). Crystal Chemistry, Nauka, Moscow, (in Russian).

Brebrick, R.F. (1967). Non-stoichiometry in Binary Semiconductor Compounds. Progress Solid State Chem., Vol. 3, No 3, (1967), pp. 213-264, ISSN 0079-6786.

Brebrick, R.F. (1963). Deviations from stoichiometry and Electrical Properties in SnTe. J. Phys. Chem. Solids, Vol. 24, No 1, (January, 1963), pp. 27-36, ISSN 0022-3697. 
Brebrick, R.F., \& Strauss, A.I. (1964) Partial Pressures in Equilibrium with Group IV Tellurides. II. Tin Telluride. J. Chem. Phys., Vol. 41, No 1, (January, 1964), pp. 197205, ISSN 0021-9606.

Brebrick, R.F. (1971). Composition stability limits for the Rocksalt-Structure Phase $\left(\mathrm{Pb}_{1-\mathrm{y}} \mathrm{Sn}_{\mathrm{y}}\right)_{1-\mathrm{x}} \mathrm{Te}_{\mathrm{x}}$ from Lattice Parameter Measurements. J. Phys. Chem. Solids., Vol. 32, No 3, (March, 1971), pp. 551-562, ISSN 0022-3697.

Brebrick, R.F., Harman, T.C., \& Strauss, A.J. (1962). Experimental investigation of band structure of SnTe. Bull. Am. Phys. Soc. ser. II., Vol. 7, No. 2, (February, 1962), pp. 203, ISSN 0003-0503.

Brebrick, R.F., \& Strauss, A.J. (1963). Anomalous thermoelectric power as evidence for twovalence bands in SnTe. Phys. Rev., Vol. 131, No. 1, (July , 1963), pp. 104-110, ISSN 1943-2879.

Bokshtein, B.S., Bokshtein, S.Z, \& Zhukhovitskii, A.A. (1974). Thermodynamics and kinetics of diffusion in solids, Metalurgiya, Moscow, (in Russian).

Brilson, L.J., Burstein, E., \& Muldawer, L. (1974). Raman observation of the ferroelectric phase transition in SnTe. Phys. Rev. B, Vol. 9, No. 4, (February, 1974), pp.1547-1551, ISSN 1098-0121.

Brukhanov, V.A., Delyagin, N.N., Kuzmin, R.I. \& Shpinel', V.S. (1964). Mossbauer effect in the binary compounds of tin. JETF, Vol. 46, No. 6, (June, 1964), pp. 1996-2001, ISSN 0044-4510.

Bukchpan, S. (1968). Determination of the Debye temperature of SnTe using the Mossbauer effect in ${ }^{119}$ Sn and ${ }^{125}$ Te. Solid State Commun, Vol. 6, No 7, (July , 1968), pp. 477-478, ISSN 0038-1098.

Burguen, Zh., \& Lanno, M. (1985). Point defects in Semiconductors. Experimental aspects, Mir, Moscow, (in Russian).

Cohen, M.L. (1961). Energy bands in the Bismuth structure. 1 A nonellipsoidal model for electrons in Bi. Phys. Rev., vol. 121, No. 2, (January, 1961), pp. 387-395, ISSN 1943-2879.

Collongues, R. (1974). Nonstoichiometry. Inorganic materials of variable composition, Mir, Moscow, (in Russian).

Collongues, R. (1993). Nonstoichiometry in ternary oxides and materials science. Jpn. J. Appl. Phys., Vol. 32, No. 3, (March, 1993), pp. 442-447, ISSN 0021-4922.

Damon, D.H. (1966). Thermal Conductivity of SnTe between $100^{\circ}$ and $500^{\circ} \mathrm{K}$. J. Appl. Phys., Vol. 37, No. 8, (July, 1966), pp. 3181-3190, ISSN 0021-8979.

Drabl, J. \& Goldsmit, G. (1963) Thermal conductivity of semiconductors, Izd. Inostr. Lit., Moscow, (in Russian)

Dzyubenko, N.I., Rogacheva, E.I., Kosevich, V.M., Laptev, S.A., \& Arinkin, A.V. (1983). The influence of indium, gallium, antimony, and bismuth on the properties of tin telluride. Izv AN SSSR, Neorgan Mater, Vol. 19, No. 9, (September, 1983), pp. 1457-1461.

Dzyubenko, N.I., Kosevich, V.M., Ob'edkov, A.G., \& Rogacheva, E.I. (1981). The behavior of the IV group elements in tin monotelluride and alloys on its basis. Izv AN SSSR, Neorgan Mater., Vol. 17, No. 1, (January, 1981), pp. 34-38.

Efimova, B.A., Kaydanov, V.I., Moyzhes B.Ya. \& Chernik, I.A. (1965). About SnTe band tructure. Fizika tverdogo tela, vol. 7, No. 10, (October, 1965), pp. 2524-2527, ISSN 0367-3294.

Erasova, N.A., \& Kaydanov, V.I. (1968). Investigation of the tin telluride thermal conductivity. Fizika i Tekhnika Poluprovodnikov, Vol. 2, No. 1, (January, 1968), pp. 34-36.

Fano, V., Fedeli, G., \& Ortalli, J. (1977). Phase transition in SnTe by Mossbauer spectroscopy. Sol. St. Communs., Vol. 22, No. 7, (May, 1977), pp. 467-470, ISSN 0038-1098. 
Flinn, P.A. (1978). Tin isomer shifts, In: Mossbauer Isomer Shifts, Shenoy, G.K., Wagner, F.E., North Holland, Amsterdam.

Fukui, K., Inoguchi, K., Kondo, S., \& Tatsukawa, T. (1984). Amorphous-crystalline transformation of SnTe thin films. Jpn. J. Appl. Phys., vol. 23, No. 8, (August, 1984), pp. 1141-1143, ISSN 0021-4922.

Houston, B.B., Allgaier, R.S., Babiskin, J., \& Siebenmann, P.G. (1964). Relationship between real and nominal carrier concentrations in p-type SnTe. Bull. Am. Phys. Soc., Vol. 9, No. 1, 2, (January, 1964), pp. 60, ISSN 0003-0503.

Glazov, L.M., \& Pavlova, L.M. (1988). Chemical Thermodynamics and Phase Equilibrium. Mettalurgia, Moscow, (in Russian).

Grassie, A.D. C., Agapito, J.A., \& Gonzalez, P. (1979). Anomalous resistivity at the structural phase transition of polycrystalline SnTe. J. Phys. C, Vol. 12, No. 24, (December, 1979), pp. L925-L927, ISSN 0022-3719.

Gorelik, S.S. \& Dashevskii, M.Ya. (1988). Materials science of semiconductors and isolators, Metalurgiya, Moscow, (in Russian).

Gorne, G.V., Zhigareva, N.K., Ivanova, A.B., \& Rogacheva, E.I. (1987). Chemical interaction in SnTe-based solid solutions in the Sn-In-Te system, Izv. AN SSSR. Neorgan.Mater., Vol. 23, No.8, (August, 1987), pp. 1152-1156.

Gornik, E., Heinrich, H., \& Palmetshofer L. (Eds.) (1982). Physics of narrow gap semiconductors, Springer, ISBN-10: 3540111913, Berlin.

Grigorovich, V.K. (1976). Hardness and microhardness of metals, Nauka, Moscow, (in Russian).

Gul'tyaev, P.V., \& Petrov, A.V. (1951). Heat capacity of some AIVBVI semiconductors. Fizika tverdogo tela, Vol. 1, No. 3, (March, 1951), pp. 1368-1370, ISSN 0367-3294.

Ioffe, A.V., \& Ioffe, A.F. (1954). Some regularities in the values of semiconductor thermal conductivity. Dokl. Akad. Nauk SSSR. Ser. Neorgan. Mater., Vol. 97, No. 5, (May, 1954), pp. 821-822.

Iisumi, M., Hamaguchi, Y., Komatsubara, K.P., \& Kato, Y. (1975). Phase transition in SnTe with low carrier concentration. J. Phys Soc. Jap., Vol. 38, No. 2, (February, 1975), pp. 443-449, ISSN 0031-9015.

Heinrich, H. (1980). Defects in IV-VI compounds. Lecture Notes in Physics, vol. 133, (1980), pp. 407-426, ISSN 0075-8450.

Julian, C.L. (1965). Theory of Heat Conduction in Rare-Gas Crystals. Phys. Rev. A, Vol. 137, No. 1A, (January, 965), pp. A128-A137 , ISSN 1050-2947.

Kafalas, J.A., Brebrick, H.F., \& Strauss, A.J. (1964). Evidence that SnTe is a Semiconductors. Appl. Phys. Letters., vol. 4, No. 5, (March, 1964), pp. 93-94, ISSN 0003-6951.

Kane, E.O. (1957). Band structure in indium antimonide. J. Phys. Chem. Solids, vol. 1, No. 4, (March, 1957), pp. 249-261.

Kaydanov, V.I., Chernik, I.A., \& Efimova, B.A. (1967). Investigation of band structure and mechanism of charge carrier scattering in SnTe. Fizika i tekhnika poluprovodnikov, Vol. 1, No. 6, (June, 1967), pp. 869-379.

Kaydanov, V.I., \& Ravich, Yu.L., (1985). Deep and resonant states in AIVBVI semiconductors. Physics-Uspekhi (Advances in Physical Sciences), Vol. 145, No. 1, (January, 1985), pp. 51-86, ISSN 0042-1294.

Katayama, S. (1976). Anomalous resistivity in structural phase transition of IV-VI compounds. Solid State Commun., Vol. 19, No. 4, (June,1976), pp. 381-383, ISSN 0038-1098.

Kattner, U., Lukas, H.L., \& Petzow, G. (1985). Optimization and Calculation of the Sn-Te system. J. Less-Common Metals., Vol. 114, No. 1, (December, 1985), pp. 129-144, ISSN 0925-8388. 
Khandozhko, A.G., Slinko, E.I., Letjuchenko, S.D., \& Tovstjuk, K.D. (1978). NMR temperature instability in SnTe. Ukrain. Phys. J., Vol. 23, (1978), pp. 1747-1749, ISSN 2071-0186.

Klemens, P.G. (1955). The Scattering of Low Frequency Lattice Waves by Static Imperfections. Proc. Phys. Soc., Vol. A68, No. 12A, (December, 1955), pp. 1113-1128, ISSN 0370-1328.

Kobayashi, K.L.I., Kato, Y., Katayama, Y., \& Komatsubara, K.P. (1975). Resistance anomaly due to displacive phase transition in SnTe. Solid State Commun., Vol. 17, No. 7, (October, 1975), pp. 875-878, ISSN 0038-1098.

Kobayashi, K.L., Kato, Y., Katayama, Y., \& Komatsubara, K.F. (1976). Carrier-concentrationdependent phase transition in SnTe. Phys. Rev. Letters, Vol. 37, No. 12, (September, 1976), pp. 772-774, ISSN 0031-9007.

Kolomoets, N.V., Laptev, S.A., \& Rogacheva, E.I. (1986). Hall factor of SnTe. Sov. Phys. Semicond., Vol. 20, No. 3, (March, 1986), pp. 283-285, ISSN 0038-5697.

Korzhuev, M.A. (1986). Germanium telluride and its physical properties, Nauka, Moscow, (in Russian).

Kröger, F.A. (1973). Chemistry of Imperfect Crystals, North-Holland, ISBN-13: 9780444105615, Amsterdam.

Lanno, M. \& Burguen, Zh. (1984). Point defects in semiconductors. Theory, Mir, Moscow, (in Russian).

Laptev, S.A. \& Rogacheva, E.I. (1986). Resonant band of tin vacancies and kinetic phenomena in SnTe. Fiz. Elektronika, Vol. 32, (1986), pp. 56-62.

Laptev, S.A. \&Rogacheva, E.I. (1988). Vacancy energy state in SnTe. Fiz. Elektronika, Vol. 36, (1988), pp. 40-45.

Lin, J.-Ch., Ngai, V., \& Chang, Y.A. (1986). Thermodynamic Properties and Defect Structure of Semiconducting Compound Phases: Tin Telluride. Metallurgical Transactions, A., Vol. 17 A, No. 7, (Jule, 1986), pp. 1241-1245, ISSN 1073-5623.

Littlewood, P.B. (1982). Phase transitions and optical properties of IV-VI compounds. Lect. Notes Phys., Vol. 152, (1982), pp. 238-246, ISSN 0075-8450.

Littlewood, P.B. (1984). Structure and bonding in narrow gap semiconductors. Crit. Revs. Solid State and Mater. Sci., Vol.11, No. 3, (1984), pp. 225-285, ISSN 1040-8436.

Logachev, Yu.A., \& Moyzhes, B.Ya. (1970). About deviation from stoichiometry in PbTe, SnTe and GeTe. Izv. AN SSSR, Neorgan.Mater., Vol. 6, No. 10, (October, 1970), pp. 1792-1797.

Maradudin, A. (1968). Defects and the vibrational spectrum of crystals, Mir, Moscow, (in Russian).

Maselsky, R., \& Lubell, M.S. (1963). Nonstoichiometry in some Group IV Tellurides. Advanc. Chem. Ser., Vol. 39, No. 2, (January, 1963), pp. 210-217, ISSN 0065-2393.

Masharov, S.I. (1963). Frequency spectrum of acoustic vibrations of crystals with vacancies. Izvestiya vuzov, No. 5, (May, 1963), pp. 39-42 , ISSN 1573-9228.

Merzhanov, L.A. (1988). Optimization of data on thermodynamic properties and tintellurium phase diagram. Vestnik Moskovskogo Univ., Ser.Khimiya, Vol. 29, No. 5, (September-October, 1988), pp. 471-474, ISSN 0579-9384.

Minemura, C., \& Morita, A. (1978). Electrical resistivity anomaly in the vicinity of structural phase transition of p-SnTe. Solid State Commun., Vol. 28, No 3, (October, 1978), pp. 273-275, ISSN 0038-1098.

Mogilevskii, V.M., \& Chudnovskii, A.F. (1972). Thermal conductivity of semiconductors, Nauka, Moscow, (in Russian). 
Mycielski, T. (1986). Formation of supperlattice of ionized resonant donors or acceptors in semiconductors. Solid State Commun., Vol. 60, No. 2, (October, 1986), pp. 165-168, ISSN 0038-1098.

Nashchekina, O.N., Rogacheva, E.I., Shpakovskaya, L.P., Pinegin, V.I., \& Fedorenko, A.I. (1995). Nonstoichiometric defects in semiconductor SnTe thin films. Mat. Res. Soc. Symp. Proc., Vol. 378, No. 2, pp. 255-260, ISSN 0272-9172.

Nashchekina, O.N., \& Rogacheva, E.I. (1998). Temperature anomalies of transport properties in SnTe epitaxial thin films. Phys. Stat. Sol. A, Vol. 169A, No. 2, (October, 1998), pp. 235-238, ISSN 1521-3951.

Nashchekina, O.N., Rogacheva, E.I., Fedorenko, A.I., \& Shpakovskaya, L.P. (1999a). Preparation and study of SnTe thin films. Functional Materials, Vol. 6, No. 2, (March-April, 1999), pp. 374-379, ISSN 1027-5495.

Nashchekina, O.N., Rogacheva, E.I., \& Fedorenko, A.I. (1999b). Phase transition in SnTe and temperature anomalies of kinetic properties. Functional Materials, Vol. 6, No. 4, (July-August, 1999), pp. 653-657, ISSN 1027-5495.

Nashchekina, O.N., Rogacheva, E.I., Fedorenko, A.I, Isakina., A.P, \& Prokhvatilov, A.I. (1999c). Low-temperature lattice instability in SnTe. Low Temperature Physics, Vol. 25, No. 4, (April, 1999), pp. 390-395, ISSN 0022-2291.

Nashchekina, O.N., Rogacheva, E.I., \& Popov, V.P. (2008). Nonstoichiometry and thermal expansion of SnTe. J. Phys. and Chem. Solids, Vol. 69, Nos 2-3, (February-March, 2008), pp.273-277, ISSN 0022-3697.

Nimtz, G., \& Schlicht, B. (Eds). (1985). Narrow-gap semiconductors, Springer, ISBN-13: 9780387120911, Berlin.

Novikova, S.I. (1974). Thermal expansion of solids, Nauka, Moscow (in Russian).

Novikova, S.I. \& Shelimova, L.E. (1965). Phase transition in SnTe. Fizika tverd. tela, vol. 7, No. 8, (August, 1965), pp. 2544-2545, ISSN 0367-3294.

Novikova, S.I. \& Shelimova, L.E. (1967). Low-temperature phase transition in SnTe. Fizika tverd. tela, vol. 9, No. 5, (May, 1967), pp. 1336-1338, ISSN 0367-3294.

Parada, N.J., \& Pratt, G.W. (1969). New Model for vacancy states in PbTe. Phys. Rev. Lett. vol. 22, No. 5, (February, 1969), pp. 180-182, ISSN 0031-9007.

Rabenau, A. (Ed.). (1975). Problems of nonstoichiometry, Metallurgy, Moscow, (in Russian).

Rabii, S. (1969). Energy-band structure and electronic properties of SnTe. Phys. Rev., vol. 182, No. 3, (June, 1969), pp. 821-828, ISSN 1943-2879.

Ravich, Yu.I., Efimova, B.A., \& Smirnov, I.A. (1970). Semiconducting Lead Chalcogenides, Plenum Press, ISBN-13: 9780306304262, New York.

Rogacheva, E.I., Dzubenko, N.I., Laptev, S.A., Kosevich, V.M., \& Ob'edkov, A.G. (1983). Effect of indium on properties of SnTe with different degree of deviation from stoichiometry. Izv. AN SSSR. Neorgan. Mater., Vol. 19, No. 4, (March, 1983), pp. 573577.

Rogacheva, E.I., Ivanova, A.B., Zhigareva, N. K., \& Ob'edkov, A.G. (1985). Effect of thermal treatment on mechanism of impurities dissolving in compounds of the $\mathrm{A}^{\mathrm{IV}} \mathrm{BVI}^{\mathrm{VI}}$ type. Izv. AN SSSR. Neorgan. Mater., Vol. 21, No. 12, (December, 1985), pp. 1762-1764.

Rogacheva, E.I., Gorne, G.V., Laptev, S.A., Arinkin, A.V., \& Vesene, T.B. (1986). Concentration dependences of properties in the SnTe homogeneity region. Izv. AN SSSR. Neorgan. Mater., Vol. 22, No. 1, (January, 1986), pp. 34-37.

Rogacheva, E.I., \& Dzyubenko, N.I. (1986). Solubility of impurities in nonstoichiometric SnTe. Izv. AN SSSR. Neorgan. Mater., Vol. 22, No. 5, (May, 1986), pp.760-761. 
Rogacheva, E.I., Gorne, G. V., Laptev, S.A., Baltrunas, D.I., Moteyunas, S.V. \& Vesene, T.B. (1988). Effect of deviation from stoichiometry on the solubility of antimony in tin monotelluride. Izv. AN SSSR. Neorgan. Mater., Vol. 24, No. 6, (June 1988), pp. 786-789.

Rogacheva, E.I. (1989). Concentration-Dependent microhardness in semiconductor solid solutions. Izv. AN SSSR. Neorgan. Mater., Vol. 25, No. 5, (May, 1989), pp. 643-646.

Rogacheva, E.I., Gorne, G.V., Zhigareva, N.K., \& Ivanova, A.B. (1991a). Homogeneity region of tin monotelluride. Inorganic Materials, Vol. 27, No. 2, (February, 1991), pp.194197, ISSN 0020-1685.

Rogacheva, E.I., Gorne, G.V., Zhigareva, N.K., \& Ivanova, A.B. (1991b). Influence of thermal treatment on the solubility of indium in tin monotelluride. Inorganic Mater., Vol. 27, No. 2, (February 1991), pp. 198-202, ISSN 0020-1685.

Rogacheva, E.I., Sinelnik, N.A., Nashchekina, O.N., Popov, V.P., Lobkovskaya, T.A. (1993). Defects of non-stoichiometry and dynamic stability of SnTe crystal lattice. Acta Physica Polonica A, Vol. 84, No. 4, (April, 1993), pp. 733-736, ISSN 0587-4246.

Rogacheva, E.I. (1993). Critical Phenomena in Heavily-Doped Semiconducting Compounds. Jpn. J. Appl. Phys., vol. 32, No. 3, (March, 1993), pp. 775-777, ISSN 0021-4922.

Rogacheva, E.I., Gorne, G.V., \& Nashchekina, O.N. (1995). Deviation from stoichiometry and lattice properties of semiconducting SnTe phase. Mat. Res. Soc. Symp. Proc., Vol. 378, No. 2, (1995), pp. 107-112, ISSN 0272-9172.

Rogacheva, E.I., Seregin, P.P., \& Nashchekina, O.N. (1997). Charge state of tin atoms in the ternary semiconductor phases of $\mathrm{Sn}_{1-x} \mathrm{Ag}_{2 x} \mathrm{Te}$. Proceedings of the 11th. Intern. Conf. on Ternary and Multinary Compounds, ICMTC-11, University of Salford, 8-12 September, 1997.

Rogacheva, E.I., Sologubenko, A.S., \& Krivulkin, I.M. (1998). Microhardness of $\mathrm{Pb}_{1-\mathrm{x}} \mathrm{Mn}_{\mathrm{x}} \mathrm{Te}$ semimagnetic solid solutions. Inorg. Materials, vol. 34, No. 6, (June 1998), pp. 545549, ISSN 0020-1685.

Rogacheva, E.I., \& Laptev, S.A. (1999). Electronic properties and energy spectrum of SnTe doped with In, Proceeding of Fourth International conference on Material Science and Material Properties for Infrared Optoelectronics, ISBN-13: 9780819434913, Kiev (Ukraine), 29 September - 2 October 1998.

Rogacheva, E.I., \& Krivulkin, I.M. (2001). Isotherms of thermal conductivity in PbTe-MnTe solid solutions. Fiz. Tverd.Tela, vol. 43, No. 6, (June, 2001), pp. 1000-1003, ISSN 0367-3294.

Rogacheva, E.I., \& Krivulkin, I.M. (2002). The temperature and concentration dependences of the charge carrier mobility in PbTe-MnTe solid solutions, Semiconductors, vol. 36, No. 9, (September, 2002), pp. 966-970, ISSN 1063-7826.

Rogacheva, E.I. (2003a). Self-organization processes in impurity subsystem of solid solutions. J. Phys. Chem. Solids, vol. 64, Nos. 9-10, (September, 2003), pp. 1579-1583, ISSN 0022-3697.

Rogacheva, E.I. (2003b). Non-Stoichiometry and Problem of Heavy Doping in Semiconductor Phases. Materials Science in Semiconductor Processing, Vol. 6, Nos. 5-6, (October - December, 2003), pp. 491-496, ISSN 1369-8001.

Rogacheva, E.I., Nashchekina, O.N., Tavrina, T.V., Vekhov, Ye.O., Sipatov, A.Yu., Dresselhaus, M.S. (2003c). Non-Stoichiometry in SnTe Thin Films and Temperature Instabilities of Thermoelectric Properties. Materials Science in Semiconductor Processing, Vol. 6, Nos. 5-6, (October-December, 2003), pp. 497-501, ISSN 1369-8001.

Rogacheva, E.I., Nashchekina, O.N., \& Dresselhaus, M.S. (2003d). The Optimization of Thermoelectric Parameters when introducing impurities with variable valence, Proceedings of 22 Int. Conf. On Thermoelectrics, La Grande Motte, 17-21 August 2003. 
Rogacheva, E.I. (2005). Non-stoichiometry and properties of ternary semiconductor phases of variable composition based on IV-VI compounds. Journal of Physics and Chemistry of Solids, Special issue, vol. 66, No. 11 (November, 2005), pp. 2104-2111: Proc. Of The $14^{\text {th }}$ International Conference on Ternary and Multinary Compounds, Denver, USA,

Rogers, L.M. (1968). Valence band structure of SnTe. J. Phys. D: Appl. Phys, Vol. 1, No. 7, (July, 1968), pp. 845-852, ISSN 0003-0503.

Shelimova, L.E. \& Abrikosov, N.Kh. (1964). Sn-Te system in the region of SnTe compound. Zhurnal neorgan. khimii, Vol. 9, No. 8, (August, 1964), pp. 1879-1883, ISSN 0036-0236 (in Russian).

Sagar, A, \& Miller, R.C. (1962). Experimental investigation of band structure of tin telluride. Proceeding of International Conference on the Physics of Semiconductors, London, July, 1962.

Show, D. (Ed.) (1975). Atomic diffusion in semiconductors, Mir, Moscow, (in Russian).

Sobolev, V.V. (1981). Intrinsic energy levels in $A^{I V} B^{V I}$ compounds, Shtiintsa, Kishinev.

Stauffer, A D., \& Aharony, A. (1992). Introduction to percolation theory, Taylor \& Fransis, ISBN-13: 978-0748402533, London and Washington D.C.

Sugai, S., Murase, K., \& Kawamura, H. (1977). Observation of soft to-phonon in SnTe by Raman scattering. Sol. St. Communs, Vol. 23, No. 2, (July, 1977), pp. 127-129, ISSN 0038-1098.

Sugawara, K., Huang, C.-Y., Inoue, M., Yagi, H., \& Tatsukawa, T. (1979). Ferroelectric semiconductor SnTe: Mn EPR as a probe of phase transition. J. Phys. Soc. Japan, Vol. 47, No. 5, (November, 1979), pp. 1739-1740, ISSN 0031-9015.

Suski, T. (1985). Phase transitions and resistivity anomalies in ( $\mathrm{Pb}, \mathrm{Sn}, \mathrm{Ge}) \mathrm{Te}$ compounds. Mater. Sci., Vol. 11, No. 1, (January, 1985), pp. 3-53, ISSN 0022-2461.

Suzuki, T., Yoshinaga, H., \& Takeuchi, S. (1991). Dislocation Dynamics and Plasticity, Springer, Berlin.

Tairov, S.M., Ormont, B.F., \& Sostak, N.O. (1970). Study of the Pb-Sn-Te system near PbTeSnTe quasibinary section. Izv. Akad. Nauk SSSR. Ser. Neorgan. Mater., Vol. 6, No. 9, (September, 1970), pp. 1584-1588.

Timmesfeld, K.H., \& Elliot, R.J. (1970). Thermal expansion and volume change of crystals containing substitutional point imperfections. Physica Status Solidi B, Vol. 42, No. 2, (March, 1970), pp. 859-869, ISSN 1521-3951.

Varisov, A.Z., Rezanov, A.I., \& Sabitov, R.M. (1966). Anharmonic effects in crystals with vacancies. Izv. Vyssh. Uchebn. Zaved. Fiz., Vol. 9, No. 5, (May, 1966), pp. 147-150, ISSN 0021-3411.

Varnek, V.A., Mazalov, L.N., Sidorov, Yu.G., Kanter, Yu.O., \& Mashchanov, V.I. (1980). Peculiarities of Mossbauer effect in SnTe and $\mathrm{Pb}_{0,9} \mathrm{Sn}_{0,1}$ Te. Izv. Akad. Nauk SSSR. Ser. Neorgan. Mater., Vol. 16, No. 5, (May, 1980), pp. 931-932.

Vodoriz, O.S., Dzubenko, N.I., \& Rogacheva, E.I. (2009). Influence of copper and nonstoichiometry on SnTe structure and electrophysical properties. Visnik Lvivskogo university, Ser. Fizuchna, Vol. 44, (2009), pp. 31-38, ISSN 1024-588X.

Wagner, C., \& Schottky, W. (1930). Theorie der geordneten Mischphasen. Z. Phys. Chem., Vol. 11, No 1, (January, 1930), pp. 163-210, ISSN 0942-9352.

Wertkheim, G. (1966). The Mossbauer effect, Mir, Moscow, (in Russian).

Zhdanova, V.V. \& Kontorova, T.A. (1965). Thermal expansion of doped germanium. Fizika tverdogo tela, Vol. 7, No. 11, (November, 1965), pp.3331-3338, ISSN 0367-3294. 


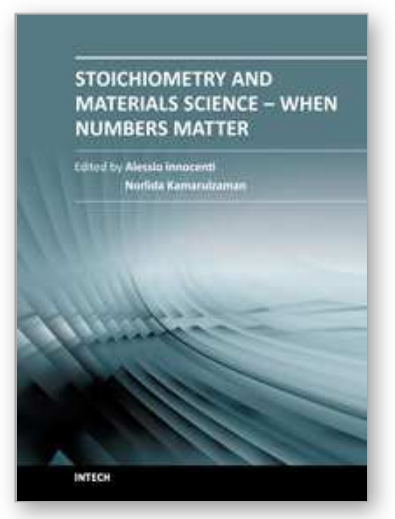

\author{
Stoichiometry and Materials Science - When Numbers Matter \\ Edited by Dr. Alessio Innocenti
}

ISBN 978-953-51-0512-1

Hard cover, 436 pages

Publisher InTech

Published online 11, April, 2012

Published in print edition April, 2012

The aim of this book is to provide an overview on the importance of stoichiometry in the materials science field. It presents a collection of selected research articles and reviews providing up-to-date information related to stoichiometry at various levels. Being materials science an interdisciplinary area, the book has been divided in multiple sections, each for a specific field of applications. The first two sections introduce the role of stoichiometry in nanotechnology and defect chemistry, providing examples of state-of-the-art technologies. Section three and four are focused on intermetallic compounds and metal oxides. Section five describes the importance of stoichiometry in electrochemical applications. In section six new strategies for solid phase synthesis are reported, while a cross sectional approach to the influence of stoichiometry in energy production is the topic of the last section. Though specifically addressed to readers with a background in physical science, I believe this book will be of interest to researchers working in materials science, engineering and technology.

\title{
How to reference
}

In order to correctly reference this scholarly work, feel free to copy and paste the following:

Elena Rogacheva (2012). Nonstoichiometry and Properties of SnTe Semiconductor Phase of Variable Composition, Stoichiometry and Materials Science - When Numbers Matter, Dr. Alessio Innocenti (Ed.), ISBN: 978-953-51-0512-1, InTech, Available from: http://www.intechopen.com/books/stoichiometry-and-materialsscience-when-numbers-matter/non-stoichiometry-and-properties-of-snte-semiconductor-phase-of-variablecomposition

\section{INTECH}

open science | open minds

\section{InTech Europe}

University Campus STeP Ri

Slavka Krautzeka 83/A

51000 Rijeka, Croatia

Phone: +385 (51) 770447

Fax: +385 (51) 686166

www.intechopen.com

\section{InTech China}

Unit 405, Office Block, Hotel Equatorial Shanghai

No.65, Yan An Road (West), Shanghai, 200040, China

中国上海市延安西路65号上海国际贵都大饭店办公楼405单元

Phone: +86-21-62489820

Fax: $+86-21-62489821$ 
(C) 2012 The Author(s). Licensee IntechOpen. This is an open access article distributed under the terms of the Creative Commons Attribution 3.0 License, which permits unrestricted use, distribution, and reproduction in any medium, provided the original work is properly cited. 